\title{
Phase Response Curve Analysis of a Full Morphological Globus Pallidus Neuron Model Reveals Distinct Perisomatic and Dendritic Modes of Synaptic Integration
}

\author{
Nathan W. Schultheiss, Jeremy R. Edgerton, and Dieter Jaeger \\ Department of Biology, Emory University, Atlanta, Georgia 30322
}

\begin{abstract}
Synchronization of globus pallidus (GP) neurons and cortically entrained oscillations between GP and other basal ganglia nuclei are key features of the pathophysiology of Parkinson's disease. Phase response curves (PRCs), which tabulate the effects of phasic inputs within a neuron's spike cycle on output spike timing, are efficient tools for predicting the emergence of synchronization in neuronal networks and entrainment to periodic input. In this study we apply physiologically realistic synaptic conductance inputs to a full morphological GP neuron model to determine the phase response properties of the soma and different regions of the dendritic tree. We find that perisomatic excitatory inputs delivered throughout the interspike interval advance the phase of the spontaneous spike cycle yielding a type I PRC. In contrast, we demonstrate that distal dendritic excitatory inputs can either delay or advance the next spike depending on whether they occur early or late in the spike cycle. We find this latter pattern of responses, summarized by a biphasic (type II) PRC, was a consequence of dendritic activation of the small conductance calcium-activated potassium current, SK. We also evaluate the spike-frequency dependence of somatic and dendritic PRC shapes, and we demonstrate the robustness of our results to variations of conductance densities, distributions, and kinetic parameters. We conclude that the distal dendrite of GP neurons embodies a distinct dynamical subsystem that could promote synchronization of pallidal networks to excitatory inputs. These results highlight the need to consider different effects of perisomatic and dendritic inputs in the control of network behavior.
\end{abstract}

\section{Introduction}

The emergence of synchronous activity in neuronal networks can result from changes in the synaptic response function of component neurons described by the phase response curve (PRC). The PRC is constructed by plotting spike time shifts caused by inputs at different times within the spike cycle. Type I PRCs for excitatory (inhibitory) stimuli are composed predominantly of positive (negative) values indicating that inputs throughout the spike cycle advance (delay) the next spike. In contrast, type II PRCs contain both positive and negative regions indicating that excitatory inputs can either advance or, paradoxically, delay the next spike depending on input phase. Type II PRCs have been related extensively in vitro and in silico to synchronization of connected neuronal networks with particular architectures (Hansel et al., 1995; Ermentrout, 1996; Crook et al., 1998a,b; Ermentrout et al., 2001; Netoff et al., 2005a,b; Goldberg et al., 2007; Achuthan and Canavier, 2009; Bogaard et al., 2009) and of uncoupled neurons receiving correlated inputs (Galán et al., 2007b; Marella and Ermentrout, 2008; Abouzeid and Ermentrout, 2009).

Recent experimental and theoretical studies have demonstrated that passive dendritic filtering of inputs and the contribu-

Received Aug. 12, 2009; revised Jan. 7, 2010; accepted Jan. 9, 2010.

This project was supported by National Institute of Neurological Disorders and Stroke Grant R01-NS039852. We thank Astrid Prinz for numerous productive discussions of PRC analysis.

Correspondence should be addressed to Dieter Jaeger, Department of Biology, Emory University, 1510 Clifton Road. NE, Atlanta, GA, 30322. E-mail: djaeger@emory.edu.

DOI:10.1523/JNEUROSCI.3959-09.2010

Copyright $\odot 2010$ the authors $\quad 0270-6474 / 10 / 302767-16 \$ 15.00 / 0$ tions of active membrane currents are critically involved in shaping the PRC and therefore network dynamics (Crook et al., 1998a,b; Gutkin et al., 2005; Goldberg et al., 2007; Stiefel et al., 2008, 2009). Sensitivity of the PRC to neuromodulation may underlie switching between different network functional states (Stiefel et al., 2008, 2009), and chronic alterations of functional network connectivity or removal of modulation are related by their effects on phase response properties to pathological network synchronization characteristic of epilepsy (Netoff et al., 2004; White and Netoff, 2008), which may similarly apply to Parkinson's disease (PD).

Synchronized oscillations and bursting in basal ganglia (BG) structures are key features of the pathophysiology of PD, and several physiological studies (Plenz and Kitai, 1999; Magill et al., 2000, 2001; Loucif et al., 2005) and network simulations (Terman et al., 2002) suggest that the globus pallidus (GP)-subthalamic nucleus (STN) feedback loop within the BG can promote oscillatory pattern generation (Bevan et al., 2002). Furthermore, recent evidence indicates an orchestrating role for GP in the $\beta$-frequency synchronization of BG activity in PD (Mallet et al., 2008). To elucidate how cellular properties of GP neurons may be involved in the emergence of synchronous states, it is important to examine what conditions can support biphasic PRCs. We used a well characterized, full morphological GP neuron model (Günay et al., 2008) to determine how PRCs of these neurons may depend on input characteristics and intrinsic cellular mechanisms. Using a model analysis allowed us to fully trace the parameter dependence of PRC shape and circumvented the ex- 
perimental problems underlying accurate PRC estimation due to intrinsic spike cycle variability, which is prominent in GP (Deister and Wilson, 2008). We found that perisomatic PRCs in GP model neurons were type I, whereas distal dendritic excitatory inputs yielded type II PRCs due to local activation of the small conductance calcium-activated potassium current (SK) at the site of stimulation. The influence of this dendritic SK mechanism on spike timing is likely to promote GP synchronization and entrainment to oscillatory STN inputs that are prominent in PD.

\section{Materials and Methods}

Simulations were run on Emory University High Performance Compute Clusters (Sun Microsystems) using the GENESIS simulation platform (www.genesis-sim.org/GENESIS). Approximately $1.5 \mathrm{~min}$ of processor time was required to simulate one second of data with $20 \mu$ s time steps using the full 585-compartment GP neuron model. Custom routines were written using Matlab (The MathWorks) for the analysis of voltage, current, conductance, and spike time data.

\section{GP neuron model}

Morphology and passive electrical properties. The morphology and passive electrical properties of our baseline GP neuron model ( $\left.\mathrm{GP}_{\text {base }}\right)$ were determined as previously described (Hanson et al., 2004; Günay et al., 2008). Briefly, the Neurolucida (MicroBrightField) reconstruction of a GP neuron that showed electrophysiological properties (spike width, spike height, input resistance, spike adaptation) typical of GP neurons in our recorded population of GP neurons (Günay et al., 2008) was converted to a GENESIS morphology file using CVAPP software (www.compneuro.org). The resultant somatodendritic morphology contained a spherical soma and 511 dendritic compartments (Fig. $1 \mathrm{~A}$ ), each no more than $0.02 \lambda$ in electrotonic length. Matching of the model to experimental voltage responses was achieved by setting passive biophysical parameters as follows: specific membrane capacitance $\left(C_{\mathrm{M}}=0.024 \mathrm{~F} / \mathrm{m}^{2}\right)$, specific membrane resistivity $\left(R_{\mathrm{M}}=1.47 \Omega \mathrm{m}^{2}\right)$, and specific axial resistivity $\left(R_{\mathrm{A}}=1.74 \Omega \mathrm{m}\right)$. To allow axonal spike initiation and realistic axonal current sources and sinks, a standard axon consisting of a highly excitable axon initial segment and nodes of Ranvier separated by myelinated internode segments, was adapted from Shen et al. (1999) and attached to the soma.

Active conductances. Based on experimental evidence of their presence in GP neurons, one calcium-activated conductance and eight voltagegated membrane conductances were added to the soma and dendrite of the passive model. Because distinct electrophysiological cell types are not apparent in GP when examined with rigorous statistical tests (Günay et al., 2008), we made a single active model resembling the mean behavior of recorded GP neurons. The voltage-gated conductances were modeled using standard Hodgkin-Huxley equations and included the following channel types: fast-transient and persistent sodium currents, NaF and $\mathrm{NaP}$ (Magistretti and Alonso, 1999, 2002; Magistretti et al., 1999; Raman and Bean, 2001; Khaliq et al., 2003; Hanson et al., 2004; Mercer et al., 2007); fast and slow delayed-rectifier $\left(K_{\mathrm{dr}}\right)$ potassium currents, $K_{\mathrm{V}} 3$ and $\mathrm{K}_{\mathrm{V}} 2$ (Baranauskas et al., 1999, 2003); A-type potassium current, which we modeled as two channel populations to accurately match fast and slow

\section{B Model vs Experimentally Measured Spike Shape}
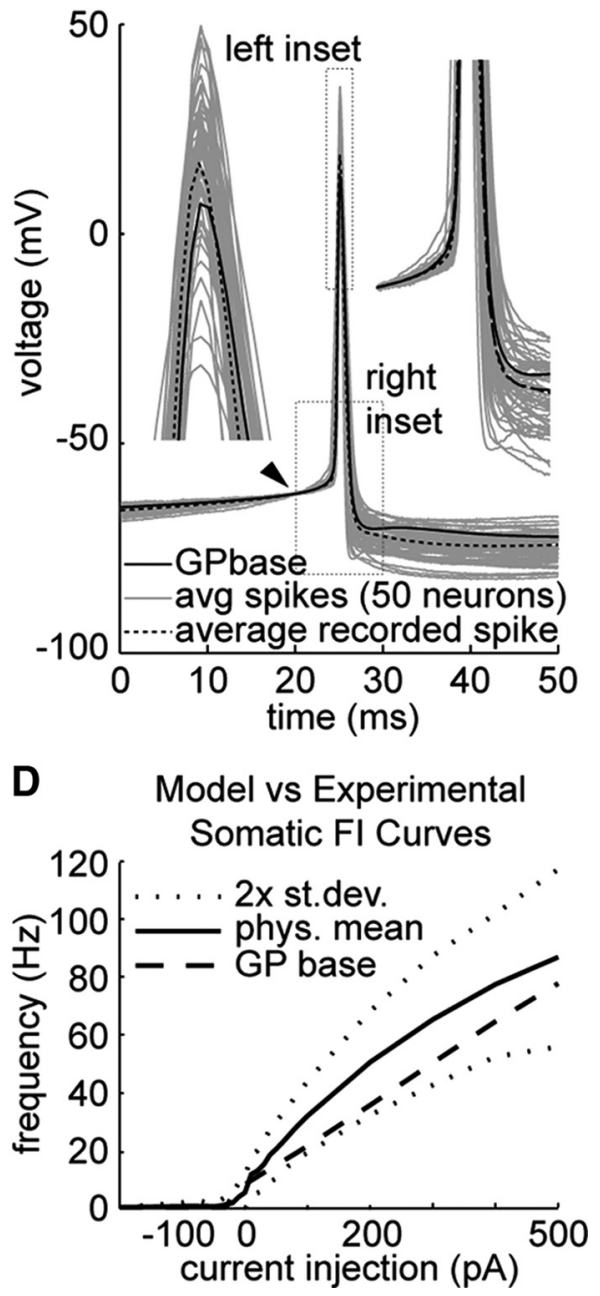

Figure 1. GP model morphology, conductances, and electrophysiological properties reproduce those of recorded GP neurons. $A$, Neurolucida (MicroBrightField) reconstruction of the GP neuron morphology used for all versions of the GP model. Somatic ( $S$ )

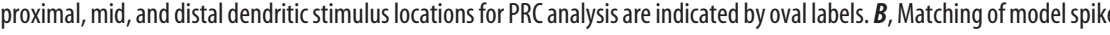
(dashed line) well within the biological variability (gray lines) for of spike onset, spike height, and spike width, and AHPs. Average spikes from 50 recorded neurons (gray lines) are anchored to the population mean voltage $5 \mathrm{~ms}$ before spike peaks (black arrowhead) to illustrate the variability of AHPs across neurons. C, Somatic voltage- and calcium-gated currents during a spontaneous action potential of $\mathrm{GP}_{\text {base }}$. Note that the $\mathrm{NaF}$ current peak is curtailed by the $x$-axis to better distinguish the other curren traces. H-current is not shown because it had minimal activation. $\boldsymbol{D}$, Frequency-current (FI) curves for the base model compared with the average experimentally measured $\mathrm{Fl}$ curve.

components of the inactivation kinetics, $\mathrm{K}_{\mathrm{V}} 4 \mathrm{~F}$ and $\mathrm{K}_{\mathrm{V}} 4 \mathrm{~S}$ (Tkatch et al., 2000); M-type potassium current, KCNQ (Gamper et al., 2003; Prole and Marrion, 2004); hyperpolarization-activated mixed cation current, or h-current, which we also modeled as two channel populations, HCN1 and HCN2 (Wang et al., 2002; Chan et al., 2004); and a high-voltageactivated calcium current $\left(\mathrm{Ca}_{\mathrm{HVA}}\right)$ representing a mixture of $\mathrm{L}-, \mathrm{N}-$, and P/Q-type currents. The calcium dependence of SK (Hirschberg et al., 1998, 1999; Xia et al., 1998; Keen et al., 1999) was modeled using the Hill equation. Table 1 in the online supplemental materials lists the activation and inactivation parameters for the conductances implemented in $\mathrm{GP}_{\text {base }}$, and supplemental Figure 1 (available at www. jneurosci.org as supplemental material) illustrates the steady-state voltage or calcium dependencies of activation and inactivation gates for each channel type.

Conductance distributions and density tuning. Each conductance was distributed uniformly throughout the dendrite with the exception of $\mathrm{Ca}_{\text {HVA }}$ whose density was greater in thinner dendritic compartments 
(Hanson and Smith, 2002). Since dendritic diameter tapered further from the soma, the highest densities of $\mathrm{Ca}_{\mathrm{HVA}}$ occurred at distal regions of dendritic branches.

During the original tuning process, somatic and dendritic conductance densities were determined using a semiautomated process comparing model behaviors with physiological recordings, and a thorough exploration of parameter space was performed (Günay et al., 2008). For this study, we updated the model to incorporate recent data describing ion channel kinetics in GP (Mercer et al., 2007) and used conductance densities within the previously explored parameter space that provided a good match with physiological current-clamp data (see supplemental Tables 1 and 2 for final parameters). The resultant base model, $\mathrm{GP}_{\text {base}}$, exhibited a spontaneous spike waveform closely matching the average spike from 50 recorded GP neurons (Fig. $1 B$ ) and fell well within the limits of physiological variability for spontaneous spike frequency (supplemental Fig. $3 A$, available at www.jneurosci.org as supplemental material), the somatic FI curve (Fig. 1D), spike frequency adaptation (SFA) and spike height attenuation (SHA) during positive current steps, voltage "sag" (a consequence of h-current activation) during negative current steps, and latency to the first spike following the offset of negative current steps (supplemental Fig. 2, available at www.jneurosci.org as supplemental material). The $\mathrm{K}_{\mathrm{V}} 2$ and $\mathrm{K}_{\mathrm{V}} 3$ conductances, in the model as in GP neurons, were the primary contributors to the delayed rectifier current (Baranauskas et al., 1999), and the peak amplitudes of the respective currents were similar (Fig. 1C). The conductance density and somatodendritic distribution of SK were the primary determinants of the depth of the mAHP, and together with $\mathrm{NaP}$ and $\mathrm{KCNQ}$, contributed significantly to the spontaneous spike frequency (supplemental Fig. $3 A$, available at www.jneurosci.org as supplemental material). Interestingly, the slope of the somatic FI curve was also influenced by the density and distribution of SK conductance (supplemental Fig. 3B, available at www. jneurosci.org as supplemental material). To determine the robustness of our PRC findings with this model, we varied key conductance parameters through a large range deviating from the base model (see Results). These manipulations also serve as explorations of potential heterogeneity among pallidal networks and of possible effects of altered modulation of GP neurons. The full GENESIS model used in this study is available for download from ModelDB (http://senselab.med.yale.edu/ModelDB/).

\section{Simulation protocols}

PRC stimulation protocol. In 72 separate simulations, inputs were delivered at each of 72 evenly distributed time points within the first interspike interval (ISI) of the control spike train. The onset of the earliest input was timed to be coincident with the somatic spike delineating the start of the spike cycle. Spike times were recorded using the GENESIS spikehistory element which provided precision of $1 \times 10^{-6} \mathrm{~s}$, and input-evoked shifts in spike timing were calculated relative to the spike terminating the first control spike cycle. PRCs were plotted as spike advances (in units normalized to the period of the control ISI) as a function of input phase, such that positive values reflected advancements of the spike cycle. Separate PRCs were constructed for somatic stimulation and for stimulation of 7 dendritic sites each composed of 25 contiguous compartments. The dendritic sites of stimulation were distributed across the three major branches of the dendrite (Fig. $1 A$ ) such that the first and second dendritic branches each had proximal and distal stimulation sites $\left(\mathrm{D} 1_{\mathrm{P} \& \mathrm{D}}\right.$ and $\left.\mathrm{D} 2_{\mathrm{P} \& \mathrm{D}}\right)$, while the longer third branch had proximal, mid, and distal stimulation sites $\left(\mathrm{D} 3_{\mathrm{P}, \mathrm{M}, \& \mathrm{D}}\right)$. To conduct PRC analyses during steadystate behavior, we let the spontaneous activity of the model settle for a $10 \mathrm{~s}$ period and used the first spontaneous spike cycle after this period to apply PRC stimulation. To avoid repeating the simulation of $10 \mathrm{~s}$ settling time many times, we saved all state variables in a "snapshot" file after conducting this simulation once, and used it for all subsequent simulations.

Inputs to the model. Stimuli were either $3 \mathrm{~ms}$ square-wave current injections or dual exponential conductance injections representing excitatory AMPAergic ( $1 \mathrm{~ms}$ rise time, $3 \mathrm{~ms}$ decay time, $E_{\mathrm{AMPA}}=0 \mathrm{mV}$ ) or inhibitory $\mathrm{GABA}_{\mathrm{A}}$ ( $1 \mathrm{~ms}$ rise time, $12 \mathrm{~ms}$ decay time, $E_{\mathrm{GABA}}=-80 \mathrm{mV}$ ) synaptic inputs. Input strength was manipulated by varying current injection amplitude or the synaptic peak conductance. Current injections or synaptic conductance inputs to dendritic regions were divided evenly among the 25 component compartments such that each received $4 \%$ of the total reported current or conductance.

Faster-spiking and higher-order PRCs. Since GP neurons in vivo spike at $\sim 30 \mathrm{~Hz}$, we drove the GP model faster than the spontaneous spike frequency by applying tonic inward current to the soma. To test how increasing spike frequency affects the PRC, we derived somatic and dendritic PRCs using $2 \mathrm{nS}$ AMPA inputs while the model spiked at specific rates between 10 and $60 \mathrm{~Hz}$. Applied current strengths necessary to achieve the desired spike frequencies $( \pm 0.5 \mathrm{~Hz})$ were determined by precise interpolation from the FI curve.

Synaptic inputs to the model, particularly the dendrite, evoked membrane currents that contributed to shifts in spike timing. In many cases the time courses of these evoked currents were not limited to the ongoing ISI, however, so it was necessary to consider multiple spike cycles to capture to full effect of an input. Effects of inputs on the timing of the second, third, and fourth subsequent spikes were calculated to give the F2, F3, and F4 PRCs, respectively. By convention (Preyer and Butera, 2005), these higher-order PRCs were plotted to the left of the primary PRC (F1). The "permanent" PRC reflects the ultimate advancement or delay of spiking measured after several spike cycles (Prinz et al., 2003a). The permanent PRCs depicted in this report were calculated by summing single-cycle PRCs F1 through F5.

Categorization of PRCs as type I or type II. The distinction between type I and type II PRCs can be somewhat ambiguous. Type I PRCs are characterized by being composed predominantly of positive values for excitatory inputs. However, there may be a small negative region early in phase corresponding to stimulus delivery during the down-stroke of an action potential, without disqualifying the corresponding PRC as type I (Rinzel and Ermentrout, 1998; Oprisan and Canavier, 2002). Using a morphological model compounds this ambiguity, because backpropagating action potentials in the dendrite are wider than somatic spikes, and dendritic stimuli lead to a correspondingly wide negative region in the PRC. To distinguish these effects from dendritic mechanisms responsible for significant delays of spike timing, we limited categorization of PRCs as type II to cases where delays of spike timing were not the consequence of direct interactions between PRC stimuli and somatic or back-propagating dendritic spikes. This was accomplished by categorizing PRCs using a variant of the $r$-value convention of Tateno and Robinson (Tateno and Robinson, 2007) as follows: The $r$ value of a PRC, a measure of how biphasic the PRC is, was defined for this study as the ratio of the negative area of the PRC (absolute value) to the positive area of the PRC (or the inverse of that ratio, whichever was smaller). PRCs were deemed type I when the $r$ value was $<0.175$, and PRCs were deemed type II when the $r$ value exceeded 0.175 .

Membrane potential and channel current analysis. To characterize the size and spatial decay of voltage transients in response to inputs we recorded membrane voltage at the soma, axon, and dendritic sites in each simulation. Dendritic voltages were recorded from the middle and edge compartments of each of the 7 sites of dendritic stimulation (Fig. $1 A$ ). In addition we recorded current traces for each type of membrane conductance at the soma, axon, and the 7 dendritic sites used for stimulation. Current traces described in this report as "somatic" reflect the sum of currents from the soma and hillock, and recordings of currents from any of the 7 dendritic sites reflect sums taken across the compartments composing that site.

To evaluate the contributions of membrane currents to input-evoked shifts in spike timing, we calculated "difference" or "evoked" currents by subtracting current traces from control simulations (without stimulus) from the corresponding stimulated traces. This subtraction isolated the evoked component of membrane current flows.

\section{Results}

\section{Responses to weak phasic inputs}

Much of conventional PRC theory relies on the assumption that inputs to a neuron are weak such that multiple inputs within a spike cycle result in the linear sum of the corresponding individual phase perturbations. Within the "domain of weak coupling," the PRC scales linearly in amplitude as a function of input 
strength, because two inputs arriving simultaneously have exactly twice the effect on spike timing as one input of the same strength. Thus, for sufficiently weak inputs the normalized PRC has a static shape called the "infinitesimal" PRC (iPRC). However, realistic synaptic conductances can violate the assumption of weak coupling causing the measured phase response curve to deviate from the iPRC (Acker et al., 2003; Netoff et al., 2005a). To evaluate the range of weak input strengths for which the somatic PRC scales linearly in our GP model, we applied $3 \mathrm{~ms}$ square-wave somatic current steps of varying amplitude at different phases of the spontaneous spike cycle and measured the resultant shifts in spike timing. Depolarizing current pulses elicited advances of the spontaneous spike rhythm that persisted indefinitely (Fig. $2 A, B$ ). For these inputs ( $\leqq 100 \mathrm{pA}$ ) there were no higher order effects of somatic stimuli on phase, i.e., all ISIs after the stimulated spike cycle were equal in duration to the spontaneous period (Fig. $2 \mathrm{~A}$ ). The magnitude of phase advancement varied smoothly with input phase (the PRC), showing greater sensitivity to inputs in the last quarter of the spike cycle and relative insensitivity to inputs arriving during the first half of the spike cycle (Fig. 2C). The somatic PRC was type I, because it was composed purely of positive values reflecting that depolarizing inputs at any phase of the spike cycle advanced the next spike. The positive peaks of normalized somatic PRCs obtained with stronger inputs occurred earlier in the spike cycle (Fig. 2C), indicating a deviation from linearity and thus the mathematical domain of weak coupling already at input amplitudes below 100 pA. Another nonlinearity was evident when $3 \mathrm{~ms}$ negative current injection pulses were applied: Within the domain of weak coupling, the iPRC obtained using positive current pulses should be an exact mirror image of that obtained with negative pulses. We observed that only for very small stimuli was the spike delay caused at different input phases equal and opposite to the spike advance caused by positive current pulses of the same amplitude. Hyperpolarizing stimuli of increasing amplitude yielded PRCs that scaled much more linearly than those obtained with depolarizing inputs of the same amplitudes, suggesting that even small amounts of depolarization caused the activation of nonlinear response properties. This leads to a fundamental difference in phase response properties between inhibitory and excitatory stimuli not predicted by PRC theory.

To delimit the domain of weak coupling for somatic inputs in our GP model we examined the features of the PRC that were most sensitive to input strength and used them as a means to evaluate the convergence of PRC shape to the iPRC. The peak of the somatic PRC occurred later in the spike cycle for weaker depolarizing inputs (Fig. $2 C$ ) and converged to a phase of $\sim 0.903$ for inputs of $7.5 \mathrm{pA}$ or less (Fig. $2 G$, solid black line). At this amplitude, PRCs also became symmetrical for depolarizing and hyperpolarizing stimuli (Fig. $2 H$ ), again indicating that $7.5 \mathrm{pA}$ stimulus amplitude was the upper limit for linear phase response behavior in a strict sense.

Next, we compared the effects of phasic somatic inputs on output spike timing with inputs delivered to a distal dendritic site (D3 ${ }_{\mathrm{D}}$, Fig. 1). Because the local input resistance of small dendrites is much higher than that of the soma, the voltage response to current injection of equal amplitude is greater. For example a 10 pA 3 ms stimulus at 0.5 phase resulted in a $0.4 \mathrm{mV}$ peak voltage deflection in the soma, whereas the amplitude of the local voltage deflection in the distal dendrite $\left(\mathrm{D} 3_{\mathrm{D}}\right.$ site) was $9.0 \mathrm{mV}$ in response to the same stimulus. Therefore, the domain of weak coupling for dendritic stimuli was even smaller than for the soma, and divergence for our two measures of linearity [peak shift (Fig. 2G) and peak symmetry (Fig. $2 H$ )] was seen for stimuli exceeding just 1 pA amplitude. The resultant distal dendritic iPRC was type I ( $r$ value $=0.066)($ Fig. $2 D, F$; supplemental Fig. 4 , available at www. jneurosci.org as supplemental material), but surprisingly, inputs delivered within a brief time-window just after a spontaneous spike resulted in slight delays of the subsequent action potential. These delays and the corresponding negative region early in the PRC (Fig. 2D, inset) were increased with higher input strengths (supplemental Fig. 4, available at www.jneurosci.org as supplemental material) and are analyzed in detail below.

Infinitesimal PRCs (1 pA stimuli) for the soma and 7 dendritic stimulation sites are shown in Figure 2I. More distal inputs yielded attenuated PRCs with peaks occurring earlier in phase as a partial consequence of passive filtering of inputs by the dendrite (Goldberg et al., 2007). Nonetheless, iPRCs for all regions of the GP model were type I, and they differed smoothly from one another as a function of distance of the stimulation sites from the soma.

\section{Responses to larger somatic current injections and realistic synaptic inputs}

The peak conductance of a single AMPAergic synaptic event is on the order of $250 \mathrm{pS}$ to $1 \mathrm{nS}$ producing a peak synaptic current of 15-60 pA (Hernández et al., 2006). Thus, in our GP model currents due to the activation of even a single synapse lie outside the domain of weak coupling that provides a basis for analytical mathematical treatment of PRCs. To address the question of how the input phase of physiologically realistic synaptic input affects spike timing, we delivered stronger current injections or dual exponential AMPA-type or $\mathrm{GABA}_{\mathrm{A}}$ conductance inputs to somatic and dendritic sites of the model. Like the weaker inputs discussed above, somatic current pulses of up to $300 \mathrm{pA}$ yielded type I PRCs, and the peak of the somatic PRC occurred earlier in the spike cycle for stronger depolarizing inputs (Fig. $3 A$ ). This pattern reflects: (1) that maximal sensitivity to weak somatic inputs occurs just before a spike, and (2) the theoretical limit of spike advancement for strong inputs (Fig. 3A, long-dashed black line) is reached when a spike is initiated immediately with delivery of the stimulus. Stronger somatic inputs caused the PRC to approach the limit of spike advancement over a larger range of phase. Thus, the peak of the PRC occurred earlier within the spike cycle and the region of the PRC just before the peak became increasingly steep (Fig. $3 A$ )

The phase shifts elicited by hyperpolarizing or inhibitory inputs are not bounded by a theoretical limit, since arbitrarily strong inhibitory inputs can theoretically delay subsequent spiking indefinitely. As a result, negative current injection delivered just before spike initiation resulted in maximal phase delays (Fig. $3 D$ ). PRCs resulting from negative current injection scaled nearly linearly with input strength and maintained the same qualitative shape (Fig. 3D).

AMPAergic synaptic inputs to the soma elicited a similar pattern of spike advances as did depolarizing current injections (Fig. $3 A$ ), and the spike delaying effects of somatic GABAergic inputs were similar to those of hyperpolarizing current injections to the soma (Fig. 3D). The only notable difference between peakamplitude-matched somatic PRCs obtained using synaptic inputs and those obtained using current injections was that the peaks of synaptic PRCs occurred slightly earlier in the spike cycle, both for excitation and inhibition. This effect is likely due to the gradual onset and delayed peak of synaptic inputs compared with the sharp onset of square-pulse current steps, thus giving rise to responses resembling those elicited by current steps occurring 
A
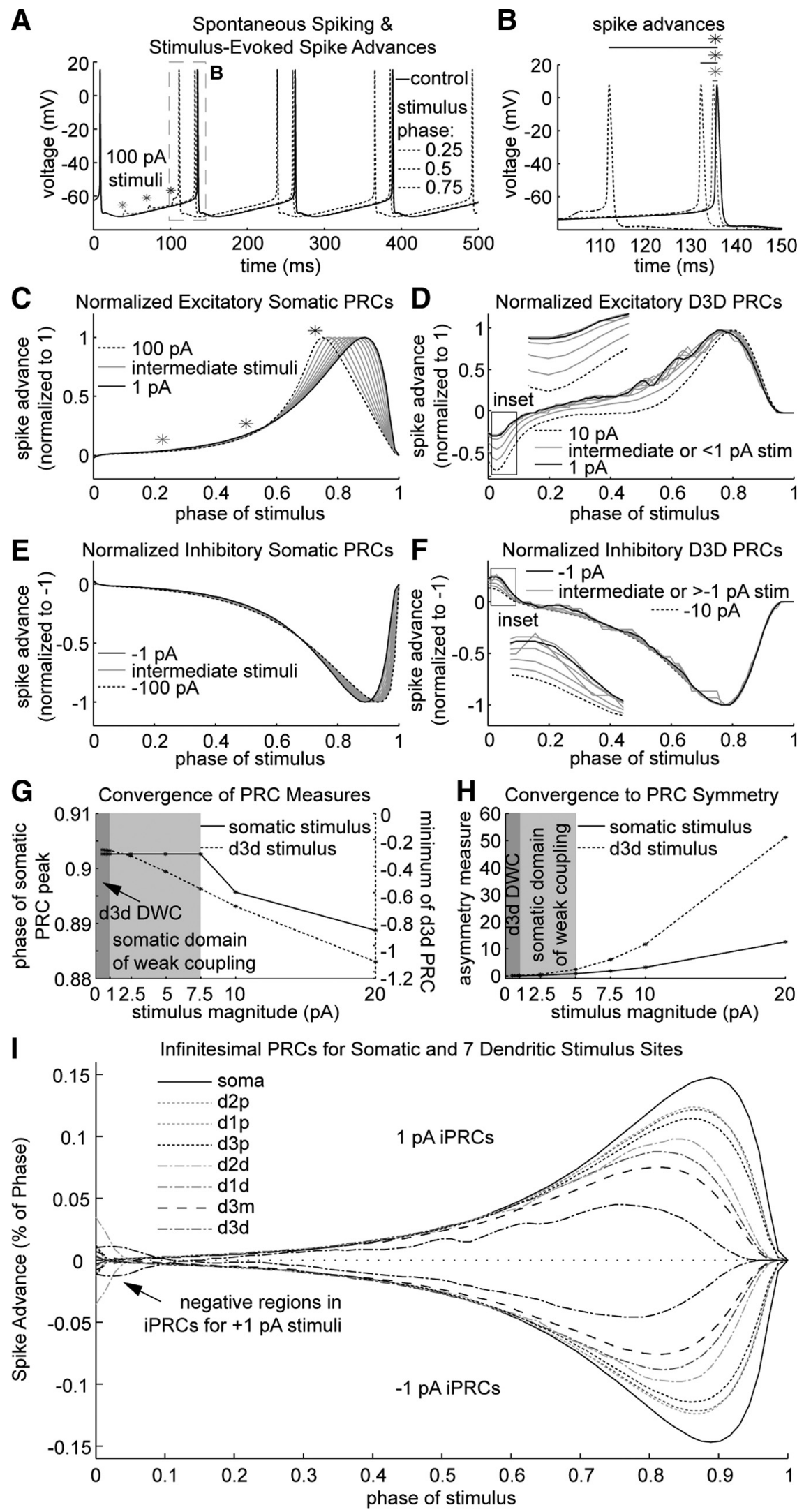

Figure 2. Physiologically realistic inputs to GP are outside somatic and dendritic domains of weak coupling. $\boldsymbol{A}$, Voltage traces illustrating spontaneous $7.9 \mathrm{~Hz}$ spiking of the model and voltage deflections and spike advances elicited by $100 \mathrm{pA}$ somatic stimuli delivered at one of three points in phase (indicated by asterisks). Note, spike advances persist indefinitely (3 spike cycles shown). $\boldsymbol{B}$, Expansion of A highlighting spike advances resulting from stimuli delivered at different phases. The earliest stimulus (indicated in $\boldsymbol{A}$-C by the lightest gray asterisk) yields the smallest spike advance, and the latest (black asterisk) yields the largest. $\boldsymbol{C}$, Normalized PRCs for somatic current injections between $1 \mathrm{pA}$ (solid black line) and $100 \mathrm{pA}$ (dotted black line). Larger somatic stimuli yield slightly later in phase. GABAergic inputs of $0.7 \mathrm{nS}$ to $2.9 \mathrm{nS}$ yielded somatic PRCs that matched in amplitude the somatic PRCs obtained using current injections of $-50 \mathrm{pA}$ to $-300 \mathrm{pA}$, whereas AMPAergic inputs of $0.5 \mathrm{nS}$ to $4.2 \mathrm{nS}$ were necessary to match the peaks of somatic PRCs for +50 $\mathrm{pA}$ to $+300 \mathrm{pA}$ current injections (Fig. $3 A, D$; supplemental Fig. $5 A, B$, available at www.jneurosci.org as supplemental material). Overall, we found good agreement between stimuli using current step inputs or conductance waveform inputs, indicating that probing PRCs with brief current steps does not limit the validity of PRC analysis.

\section{Type II PRCs from stronger, more distal dendritic inputs}

Globus pallidus neurons have long, thin dendrites that create a flat disc-like field up to $1 \mathrm{~mm}$ wide and oriented perpendicular to striatal inputs (Park et al., 1982; Yelnik et al., 1984; Kita and Kitai, 1994). Nonuniform dendritic distributions of membrane conductances (Hanson and Smith, 2002; Hanson et al., 2004) and higher local input resistance at thinner, more distal dendritic segments make it

\footnotetext{
PRCs with peaks occurring earlier in phase. The shape of normalized somatic PRCs converges for stimuli weaker than 7.5 pA. $\boldsymbol{D}$, Normalized PRCs derived by stimulating the distal region of dendrite $3\left(D 3_{D}\right)$ with current pulses between $0.5 \mathrm{pA}$ and $10 \mathrm{pA}$ (dotted black line). The shape of normalized $\mathrm{D}{ }_{\mathrm{D}}$ PRCs converges for stimuli $\leq 1 \mathrm{pA}$ (solid black line) with an $r$-value of 0.0675 indicating that the iPRC for D3 ${ }_{D}$ is type I. This can be seen most readily by examining the negative peaks of these PRCs occurring at a phase of $\sim 0.025$ (box and inset). $\boldsymbol{E}$, Normalized PRCs for negative somatic current injections between $-1 \mathrm{pA}$ (solid black line) and $-100 \mathrm{pA}$ (dotted black line). Larger negative stimuli yield PRCs with more rightshifted peaks. Note by comparison to C, $1 \mathrm{pA}$ and $-1 \mathrm{pA}$ somatic PRCs are symmetrical across zero. $F$, Normalized PRCs for $D 3_{D}$ current injections between $-0.5 \mathrm{pA}$ and $-10 \mathrm{pA}$ (dotted black line). The shapes of these normalized D ${ }_{D} P R C s$ converge for stimuli of or greater than $-1 \mathrm{pA}$ (solid black line). This can be seen most readily by noting the positive peaks of PRCs at a phase of $\sim 0.025$ (box and inset). G, Domains of somatic and $D 3_{D}$ weak coupling illustrated by plotting the relationships between features of the PRCs and stimulus strength. For somatic current injections of $7.5 \mathrm{pA}$ or less, the phase at which the peak of PRCs occurs (left $y$-axis) has converged. For D $3_{D}$ current injections of $1 \mathrm{pA}$ or less, the negative peak of PRCs (right $y$-axis) has converged. $\boldsymbol{H}$, Domains of somatic and D $3_{D}$ weak coupling illustrated by plotting a measure of PRC asymmetry (obtained by summing together PRCs for positive and negative current injections of equal magnitude at all points in phase) against stimulus strength. Excitatory somatic PRCs for stimulus strengths $>5 \mathrm{pA}$ are increasingly asymmetric with inhibitory somatic PRCs of equal strengths. Excitatory D3 ${ }_{D}$ PRCs for stimulus strengths $>1 \mathrm{pA}$ are increasingly asymmetric with inhibitory D3 ${ }_{D}$ PRCs of equal strengths. I, Infinitesimal PRCs (1 pA and $-1 \mathrm{pA}$ ) for all stimulus locations are type $\mathrm{I}$.
} 

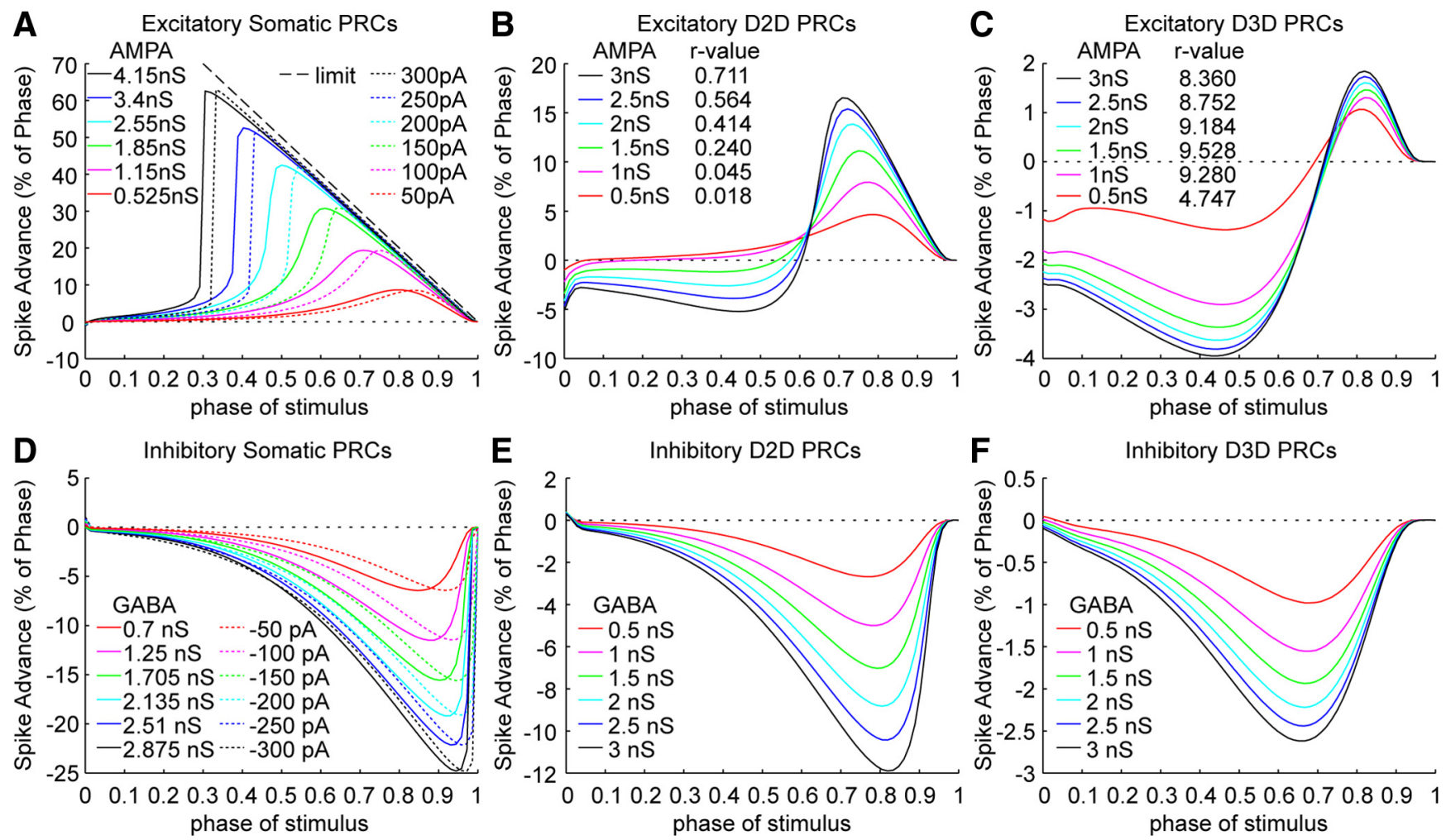

Figure 3. PRC shape depends both on stimulus location and strength. $\boldsymbol{A}$, Somatic PRCs are always type I for square-pulse positive current injections and for simulated excitatory synaptic AMPA conductances. Somatic PRCs have peaks that scale approximately linearly with stimulus magnitude, and larger stimuli cause more of the late-phase PRC to approach the limit of spike advancement. $B$, Small excitatory synaptic inputs to the end of a shorter dendrite $\left(D 2_{D}\right)$ yield type I PRCS, however, larger inputs to $D 2_{D}$ yield PRCS of increasing type II character. Both positive and negative peaks of $D 2_{D} P R C$ increase in size with stimulus strength. $C$, Excitatory synaptic inputs to the end of the longest dendrite (D $3_{D}$ ) yielded type II PRCs for all input strengths tested in this set of experiments.

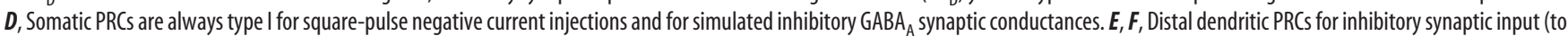
$D 2_{D}$ in $\boldsymbol{E} ;$ to $D 3_{D}$ in $\boldsymbol{F}$ ) are type I.

unlikely that inputs to different sites along the length of GP dendrites will evoke equivalent voltage transients. To test how the strength and timing of physiologically realistic inputs to different sites of the dendritic tree affect output spike timing, we constructed PRCs for seven dendritic sites using synaptic inputs of a range of peak conductance amplitudes. In each case PRCs for proximal dendritic inputs were qualitatively similar to somatic PRCs (data not shown). Surprisingly, larger AMPAergic inputs to the distal sites of the first and second dendrites and to the middle of the longest dendrite $\left(\mathrm{D} 1_{\mathrm{D}}, \mathrm{D} 2_{\mathrm{D}}\right.$, and $\left.\mathrm{D} 3_{\mathrm{M}}\right)$ yielded PRCs with increasing type II character. (PRCs for $\mathrm{D} 2{ }_{\mathrm{D}}$ are shown in Fig. $3 B$, and $r$ values quantifying the increase in type II character for stronger synaptic inputs are plotted in supplemental Fig. 6A, available at www.jneurosci.org as supplemental material.) Whereas D2 ${ }_{D}$ PRCs for excitatory inputs of $0.5 \mathrm{nS}$ and $1 \mathrm{nS}$ were type I ( $r$ values of 0.0183 and 0.0449 , respectively), inputs stronger than $1 \mathrm{nS}$ significantly delayed the next spike when delivered any time during the first half of the spike cycle. For AMPAergic inputs of $1.5 \mathrm{nS}$ to $3 \mathrm{nS}$ to $\mathrm{D} 2_{\mathrm{D}}$, the negative regions of the corresponding type II PRCs ( $r$ values of $0.24-$ 0.71 ) were progressively deeper. Thus, paradoxically, strengthening "excitatory" synaptic input to D2 ${ }_{\mathrm{D}}$ changed the net effect of the input from phase-advancing to phase-delaying for a significant portion of the spike cycle. Increasing AMPA input strength also increased the proportion of the spike cycle for which inputs delayed the subsequent spike. The positive peaks of those PRCs were also increased for stronger excitatory inputs (Fig. $3 B$ ) resulting in biphasic, type II PRCs of increasing amplitude.

Excitatory synaptic inputs to the distal-most site of the longest dendrite $\left(\mathrm{D} 3_{\mathrm{D}}\right)$ yielded type II PRCs for all input strengths tested
( $r$ values $\geqq 4.75$ for inputs $\geqq 0.5 \mathrm{nS}$ ) (Fig. $3 C$; supplemental Fig. $6 B$, available at www.jneurosci.org as supplemental material), but as was the case for $\mathrm{D} 2{ }_{\mathrm{D}}$, stronger AMPA inputs to $\mathrm{D} 3_{\mathrm{D}}$ increased the depth of the negative region in the PRC (Fig. $3 C$ ). (Note that in Fig. 2D, current injections of as little as $10 \mathrm{pA}$ notably increased the type II character of the $\mathrm{D} 3_{\mathrm{D}}$ PRC.) A prominent step in the depth of the negative region of the $D 3_{D}$ PRC occurred between AMPA input strengths of $0.5 \mathrm{nS}$ (Fig. 3C, red line) and $1 \mathrm{nS}$ (Fig. 3C, magenta line), but strengthening AMPAergic synaptic inputs beyond $1 \mathrm{nS}$ increased the amplitude of the $\mathrm{D} 3_{\mathrm{D}}$ PRC without qualitatively affecting its shape (Fig. $3 C$; supplemental Fig. $6 B$, available at www.jneurosci.org as supplemental material). In general, inputs that were applied at distal dendritic sites shifted spike timing by smaller amounts than stimuli at more proximal sites. Comparing the $\mathrm{D} 2_{\mathrm{D}}$ and $\mathrm{D} 3_{\mathrm{D}}$ stimulation sites, the maximal spike advance for stimuli occurring late in phase was reduced $\sim 10$-fold, however, the spike delays reflected in the negative region of the PRC were of similar magnitude. This indicates that excitatory inputs even at very distal sites can contribute significantly to spike delays resulting from earlyphase excitatory inputs.

GABAergic inputs to distal sites of the three major dendritic branches, $\mathrm{D} 1_{\mathrm{D}}, \mathrm{D} 2_{\mathrm{D}}$, and $\mathrm{D} 3_{\mathrm{D}}$, yielded monophasically negative, type I PRCs for all input strengths tested (Fig. $3 E, F$ ). Thus, inhibitory inputs to the distal dendrite delivered at any time within the spike cycle delayed the next spike, and there was no dependence of the qualitative shape of the PRC on the strength of those inputs. Since increasing the strength of inhibitory inputs did not qualitatively affect PRC shape, we hypothesized that intrinsic 
A D2D Voltages
(Local to $0.5 \mathrm{nS}$ Stimulus)

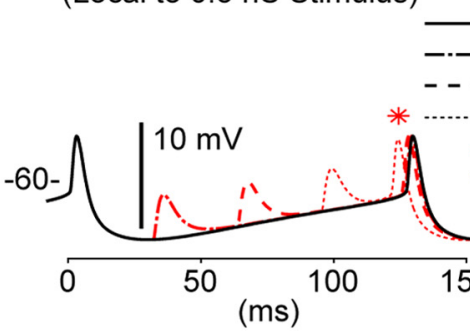

C D2D Difference Currents (Evoked by $0.5 \mathrm{nS}$ Stimulus)
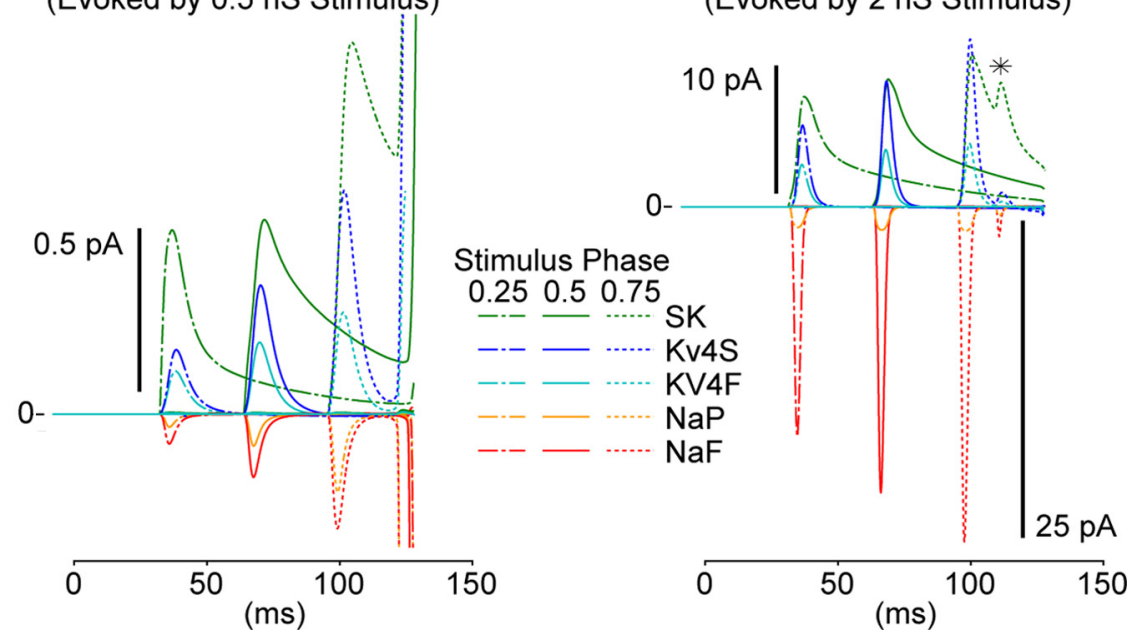

Figure 4. Different input strengths evoke different contingents of membrane currents at the distal dendrite. Shown are stimulus-evoked voltage and current transients for $0.5 \mathrm{nS}$ and $2 \mathrm{nS}$ excitatory inputs to $\mathrm{D2}{ }_{\mathrm{D}} . A$, Voltage deflections elicited by $0.5 \mathrm{nS}$ excitatory synaptic input to $D 2_{D}$ at phases of $0.25,0.5$, or 0.75 (red dashed lines, indicating phase advances). Importantly, these stimuli yield a type I D2 $2_{D} P R C$. The unstimulated voltage trajectory at $D 2_{D}$ (black trace) illustrates the back-attenuated voltage oscillation driven by spontaneous axosomatic spiking. Small spike advancements are evident in the stimulated $D 2_{D}$ voltage traces relative to the control trace (red $*$ ), particularly for the late-phase stimulus. $\boldsymbol{B}$, Voltage deflections elicited by $2 \mathrm{nS}$ excitatory synaptic input to $D 2_{D}$ at phases of $0.25,0.5$, or 0.75 (dashed blue or red lines, indicating phase delays or advances, respectively). Importantly, these stimuli yield a type II D2 $2_{D} P R C$. C, Difference currents $\left(I_{\text {Diff }}\right)$ local to $0.5 \mathrm{nS} \mathrm{D2}$ s stimuli were calculated by the subtraction of control current traces from stimulated current traces. The five largest difference currents are shown, and of these, $I_{\text {Diff-sK }}$ (green traces) is both the largest and longest lasting. Only slight differences are evident in the currents evoked by stimuli at difference phases (here, and in $\boldsymbol{D}$ ), because the control voltage trajectory at $D 2_{D}$ is relatively flat. Note that for visual clarity, $I_{\text {Diff }}$ traces in C are curtailed at the time of the next spike. D, Difference currents local to $2 \mathrm{nS} \mathrm{D2}$ stimuli. A fourfold increase in input strength (compared with $\boldsymbol{C}$ ) yields a much larger transient increase in $I_{\text {Diff-NaF }}$ which amplifies the voltage deflection (red traces) and a 20 -fold increase in peak Diff-sk $_{\text {( }}$ ( reen traces). Note that the spike advancement caused by the late-phase stimulus results in a second peak in $I_{\text {Diff-SK }}(*)$.

membrane currents activated more strongly by stronger depolarizing inputs were responsible for the increasing type II character of excitatory distal dendritic PRCs.

\section{Strong AMPA inputs to D2D evoked outward SK current in} excess of combined inward synaptic and membrane currents Increasing the strength of excitatory inputs to $\mathrm{D} 2{ }_{\mathrm{D}}$ transitioned the PRC from type I to type II in a graded manner (Fig. $3 B$ ). To evaluate which membrane currents contributed to this transition we compared the local voltage trajectories and patterns of locally evoked currents for weak and strong inputs to D2 (Fig. 4). Each panel in Figure 4 shows voltage or current transients evoked by inputs at three different times within the spike cycle. Note that the control voltage traces from $\mathrm{D} 2 \mathrm{D}_{\mathrm{D}}$ (Fig. $4 A, B$, solid black lines) contain attenuated back-propagated spikes of $\sim 5 \mathrm{mV}$ amplitude which delineate the spike cycle, and red and blue dashed traces illustrate advancements or delays of the next spike, respectively.
The gross shape of the PRC for inputs to $\mathrm{D} 2{ }_{\mathrm{D}}$ can be inferred from inspection of the local voltage trajectories relative to the control. Following the depolarizing transients evoked by $0.5 \mathrm{nS}$ AMPA input to $\mathrm{D} 2{ }_{\mathrm{D}}$, the local voltage trajectories for stimulated trials converged back toward the control trajectory, but remained slightly depolarized relative to the control until the next spike event (Fig. 4A). Thus, 0.5 $\mathrm{nS}$ AMPA input at all phases of the spike cycle resulted in spike advances (Fig. $4 A$, red traces). In contrast, following the larger depolarization transients evoked by $2 \mathrm{nS}$ AMPA inputs to $\mathrm{D} 2_{\mathrm{D}}$, the local voltage trajectories hyperpolarized below the control until the time of the next spike (Fig. 4B). For the "late-phase" stimulus, the next spike occurred almost immediately after the depolarization transient (Fig. $4 B$, red trace) corresponding to an advancement of the spike cycle. However, inputs to $\mathrm{D} 2 \mathrm{D}_{\mathrm{D}} \mathrm{de}$ livered earlier in the spike cycle resulted in delays of the subsequent spike (Fig. $4 B$, blue traces) as the local membrane remained hyperpolarized below control traces.

Figure 4, $C$ and $D$, depicts the five intrinsic membrane currents evoked most strongly by distal dendritic synaptic inputs in the stimulated compartments. Local voltage transients in response to $0.5 \mathrm{nS}$ and $2 \mathrm{nS}$ inputs to $\mathrm{D} 2{ }_{\mathrm{D}}$ were $\sim 5$ and 25 $\mathrm{mV}$, respectively (Fig. $4 A, B$ ). This supralinear relationship between input and response amplitudes was primarily due to stronger activation of inward $\mathrm{NaF}$ current by the stronger stimulus (Fig. $4 C, D$, red lines). The most pronounced effect, however, of strengthening synaptic input to the distal dendrite was the increased local activation of the small conductance calcium-activated potassium current, SK (Fig. $4 C, D$, green lines). The peak of the SK current evoked by the $2 \mathrm{nS}$ input was 15 -fold that of the $0.5 \mathrm{nS}$ input. Though the peak amplitude of the evoked SK current was smaller than the peak evoked $\mathrm{NaF}$ current, the duration of SK activation was much longer lasting, resulting in local hyperpolarization and a net current sink in response to early-phase AMPA stimulation and explaining how excitatory inputs can yield phase delays.

\section{Comparison of voltage-gated and synaptic currents explains somatic type I and dendritic type II PRCs}

Whereas D2 $2_{D}$ PRCs transitioned from type I to type II for stronger synaptic inputs, somatic PRCs were type I and D $3_{D}$ PRCs were type II over the entire physiological range of stimulus strengths tested. Figure 5, $A-D$, shows the local responses to $1 \mathrm{nS}$ AMPA inputs to the soma and $\mathrm{D} 3_{\mathrm{D}}$ allowing the comparison of voltage and current transients between morphological sites for which identical inputs yielded type I and type II PRCs, respectively. The voltage trajectories elicited by somatic inputs remained depolar- 
ized relative to the control until the next spike event (Fig. 5A). In contrast, inputs to $\mathrm{D} 3_{\mathrm{D}}$ evoked larger transients of depolarization $(\sim 40 \mathrm{mV})$ followed by deep hyperpolarizations of the local membrane (Fig. $5 B$ ), similar to the effect of strong AMPA inputs to $\mathrm{D} 2_{\mathrm{D}}$.

During the spontaneous spike cycle the somatic voltage traversed a much larger subthreshold range $(\sim 10 \mathrm{mV})$ than did the distal dendritic voltage $(<5 \mathrm{mV})$. Consequently, the contingent of currents evoked locally by somatic AMPA inputs depended more strongly on the phase of the input (Fig. $5 C$ ) than did the pattern of currents evoked by inputs to $\mathrm{D} 3_{\mathrm{D}}$. However, the somatic membrane currents evoked by $1 \mathrm{nS}$ AMPA inputs were insignificant relative to the synaptic current (Fig. $5 E$ ), because the voltage deflection caused by the stimulus was small. Therefore, somatic inputs resulted in type I PRCs, because for stimuli at all phases of the spike cycle the inward synaptic current overshadowed the net-outward contribution of evoked membrane currents (Fig. 5E).

Because action potentials did not back-propagate efficiently into the distal dendrite in our $\mathrm{GP}_{\text {base }}$ model, the control voltage trajectory at $\mathrm{D} 3_{\mathrm{D}}$ was relatively flat throughout the spike cycle (Fig. $5 B$ ). Consequently, the pattern of intrinsic currents evoked by $\mathrm{D} 3_{\mathrm{D}}$ inputs was relatively independent of input phase. AMPA inputs of 1 $\mathrm{nS}$ to $\mathrm{D} 3_{\mathrm{D}}$ evoked intrinsic membrane currents with a fast inward component and a slower outward component (Fig. $5 D, F)$, as follows: Strong, "fast" $\mathrm{NaF}$ current $(\sim 30 \mathrm{pA})$ amplified the immediate depolarization evoked by an input. In turn, higher calcium influx through the high-voltage-activated calcium channel $\left(\mathrm{Ca}_{\mathrm{HVA}}\right.$, the calcium source for SK in the model) elicited greater activation of the long-lasting, "slow" SK current (peak $>20$ pA) (Fig. 5D). As depicted in Figure $5 F$, the net outward charge transfer by local intrinsic membrane currents at $\mathrm{D} 3_{\mathrm{D}}$ was sufficiently large to exceed the depolarizing charge carried by the inward synaptic current (Fig. 5F).

\section{The type II character of distal dendritic PRCs directly reflects local SK conductance}

The analysis of membrane currents elicited locally by strong synaptic stimulation of distal dendritic sites indicated that activation of the SK conductance by calcium influx played a predominant role in sculpting type II dendritic PRCs. To test this hypothesis directly we manipulated

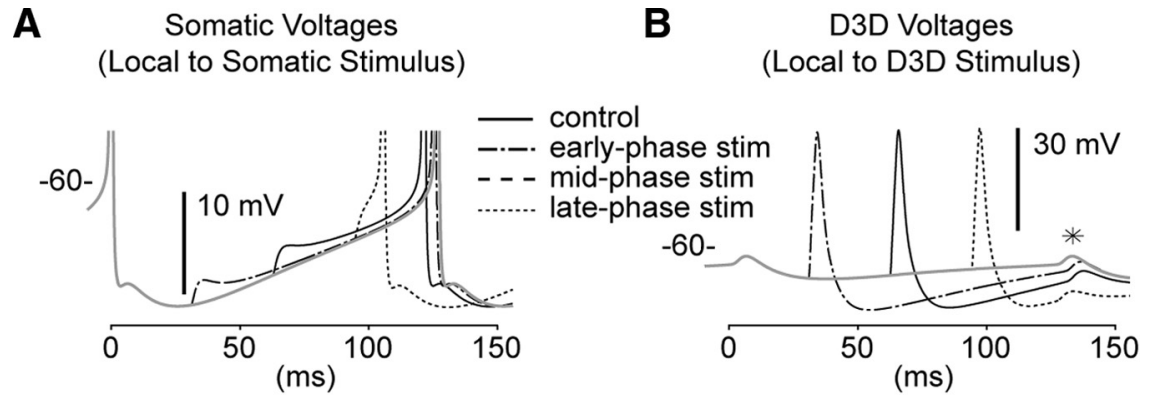

C Somatic Difference Currents

D D3D Difference Currents

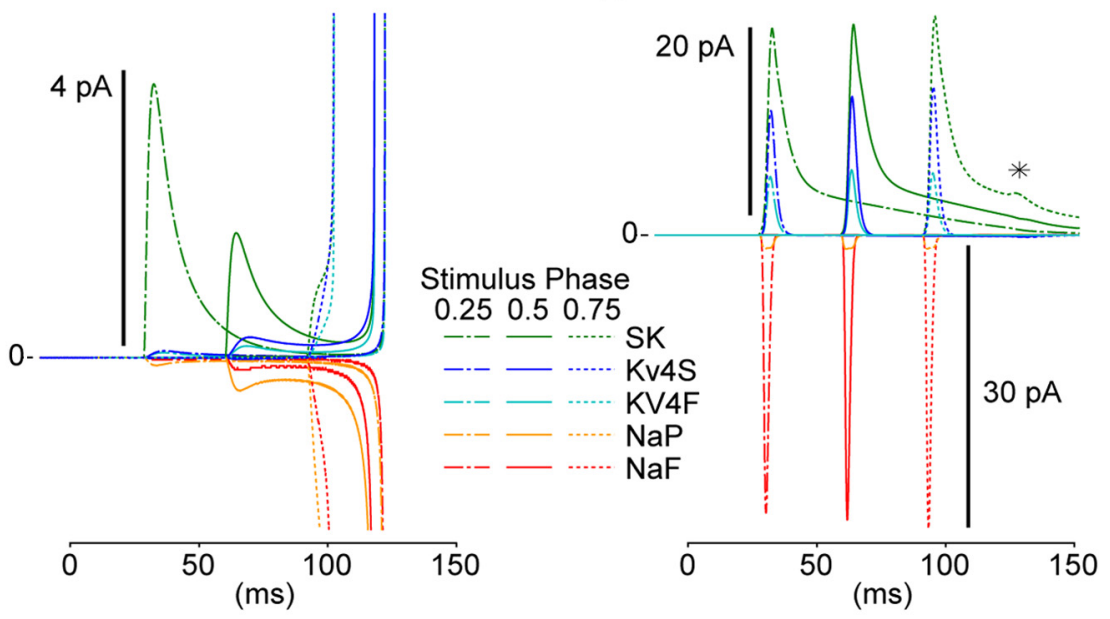

E Somatic Difference Charge Flows

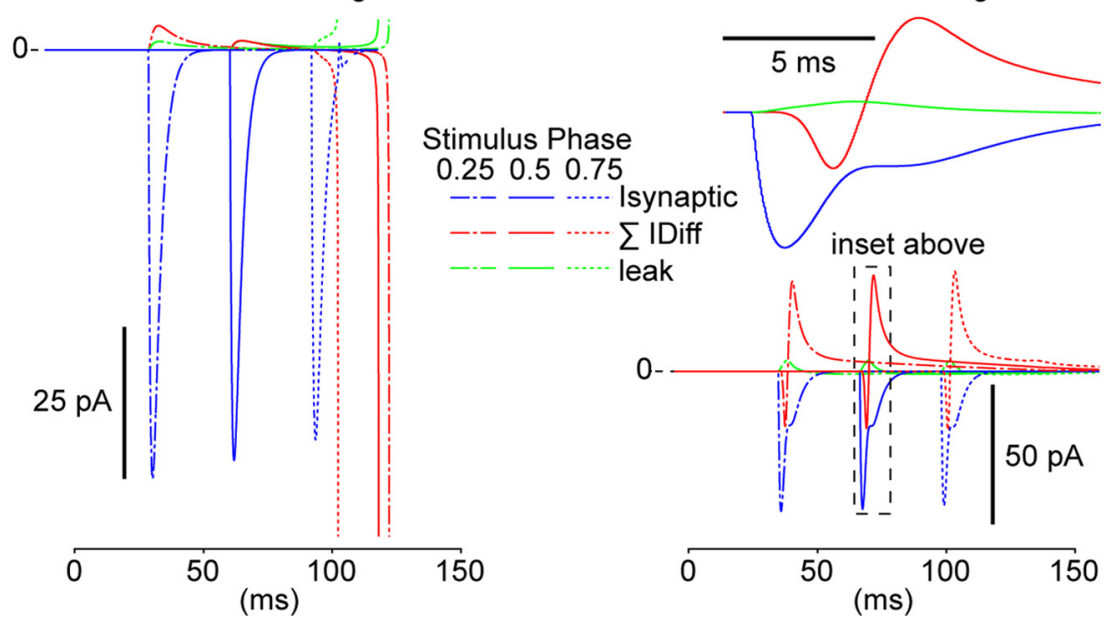

Figure 5. Targeting identical stimuli to different regions of the model evokes different contingents of membrane currents. Shown are stimulus-evoked voltage and current transients for $1 \mathrm{nS}$ excitatory inputs to the soma vs the most-distal region of dendrite, $\mathrm{D} \mathrm{B}_{\mathrm{D}} \cdot A$, Voltage deflections elicited by $1 \mathrm{nS}$ excitatory synaptic input delivered to the soma at phases of $0.25,0.5$, or 0.75 (dashed or solid black lines, as indicated). Importantly, these stimuli yielded a type I somatic PRC. The unstimulated somatic voltage trajectory (gray line) covers approximately a $10 \mathrm{mV}$ range between the depth of the AHP and spike threshold. As in Figure 4, spike advancements relative to control are evident, particularly for the late-phase stimulus. $\boldsymbol{B}$, Voltage deflections elicited by $1 \mathrm{nS}$ excitatory synaptic input to $\mathrm{D} 3_{\mathrm{D}}$ at phases of $0.25,0.5$, or 0.75 (dashed or solid black lines, as indicated) are more than 10 -fold larger than those elicited by equivalent somatic inputs, because D $3_{D}$ input resistance is much higher. Importantly, these stimuli yield a type II D3 ${ }_{D}$ PRC. Slight spike delays and advancements relative to control are again evident ${ }_{*}$ ). $C$, Difference currents local to $1 \mathrm{nS}$ somatic stimuli. The pattern of evoked currents differs depending on input phase, because the control somatic voltage trajectory is not flat. As in Figure $4, I_{\text {Diff }}$ traces are curtailed at the time of the next spike. D, Difference currents local to $1 \mathrm{nS} \mathrm{D3}$ D stimuli. D3 ${ }_{D}$ stimuli yielded a peak $I_{\text {Diff-SK }}$ (green traces) that is five times greater than that elicited by identical somatic stimuli. Minimal differences are evident in the pattern of currents evoked by stimuli at difference phases, because the control voltage trajectory at $D 3_{D}$ is flat. $\boldsymbol{E}$, The synaptic current of the stimulus overwhelms membrane currents evoked at the soma. The sum of all somatic difference currents and difference leak are negligible relative to the synaptic current of a $1 \mathrm{nS}$ AMPA input which is $\sim 70 \mathrm{pA} . \boldsymbol{F}, \mathrm{A} 1$ nS AMPA input to D3 $3_{D}$ yields a smaller synaptic current than the equivalent conductance input applied to the soma, because the $D 3_{D}$ voltage deflection is much larger, reducing the synaptic driving force. The sum of all $\mathrm{D}_{\mathrm{D}}$ difference currents, of which $\mathrm{NaF}$ and $\mathrm{SK}$ are the major contributors (D), is sufficiently large (and long-lasting) to rival the synaptic current. 
A

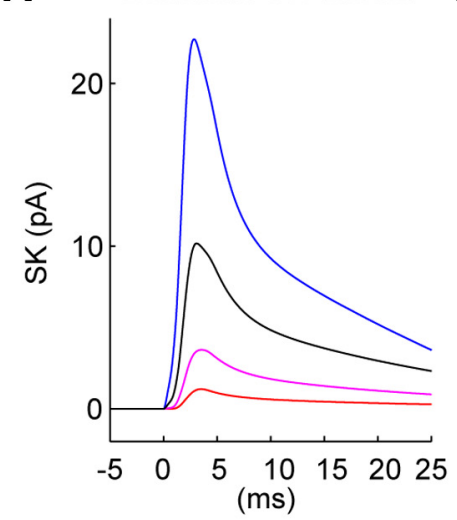

2xHVA, $2 \times S K$

1XHVA, 1xSK

C

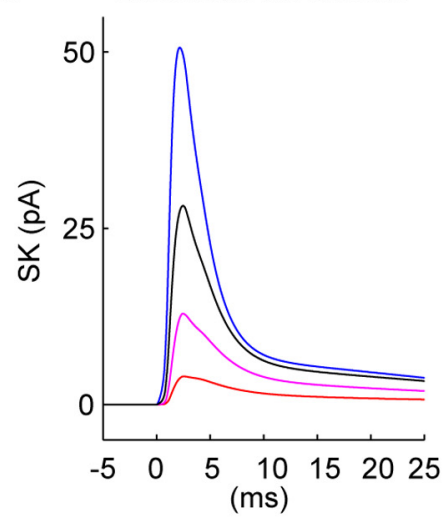

2xHVA, $2 x S K$ $1 \mathrm{XHVA}, 1 \times \mathrm{XSK}$
B

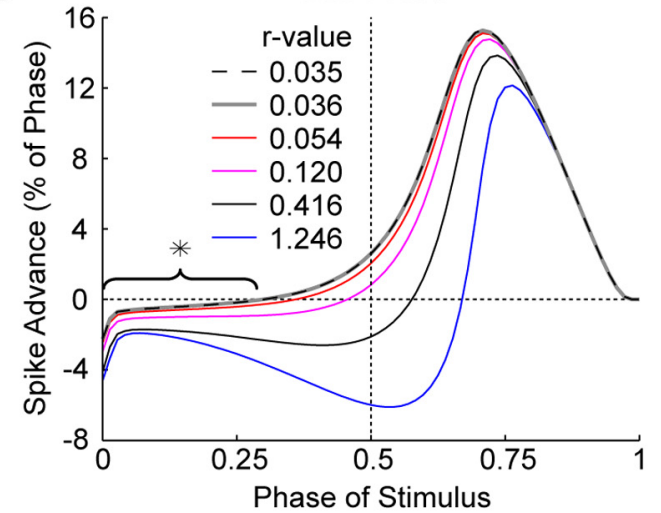

0.5xHVA, 0.5xSK $0.25 x H V A, 0.25 \times S K$
D2D PRCs

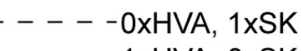
1XHVA, 0xSK
D

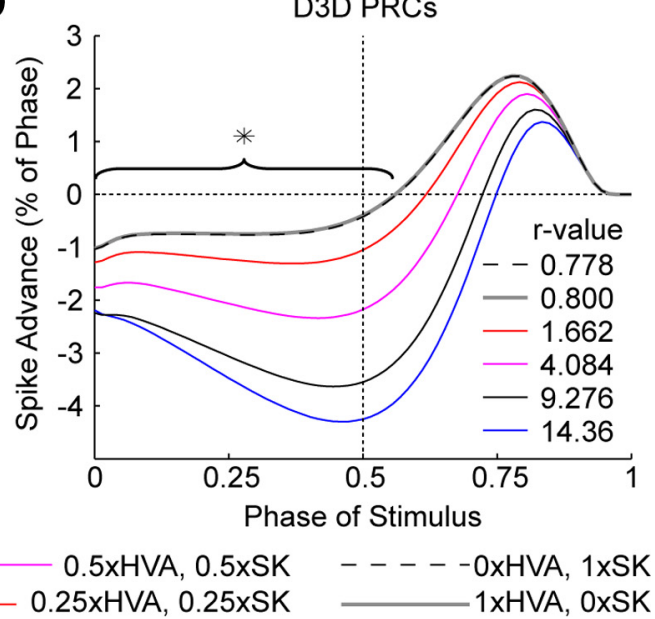

Figure 6. Tandem local up- and downregulation of $\mathrm{Ca}_{\mathrm{HVA}}$ and SK modulates the depth of the negative peak of distal dendritic PRCS. A, SK difference currents evoked by $2 \mathrm{nS}$ excitatory synaptic input to $\mathrm{DZ}_{\mathrm{D}}$ in models where the densities of $\mathrm{Ca}_{\mathrm{HVA}}$ and $\mathrm{SK}$ have been dialed up or down in the compartments composing the $\mathrm{D}_{D}$ stimulus site. (Note that since the manipulation of conductance densities was limited to the 25 compartments of the $D 2_{D}$ site, the spontaneous spiking behavior of the model was unaffected.) Traces are aligned such that stimulus onsets are at zero. $B, D 2_{D}$ PRCs transition from type I to type Il as $\mathrm{Ca}_{\mathrm{HVA}}$ and $S K$ are upregulated local to stimulus delivery. Visually identical type I PRCs result when either $\mathrm{C}_{\mathrm{HVA}}$ or $S K$ are eliminated from $D 2_{D}$ (gray and dashed black lines). The negative region of $\mathrm{D}_{\mathrm{D}}$ PRCs (asterisk) that remains when the $\mathrm{C}_{\mathrm{HVA}} / \mathrm{SK}$ mechanism had been locally disabled (by eliminating either $\mathrm{Ca}_{\mathrm{HVA}}$ or SK) stems from compartments neighboring $\mathrm{D} 2_{\mathrm{D}}$ where the mechanism was left intact and that also experience significant voltage deflection evoked by the stimulus. $C$, $\mathrm{Ca}_{\mathrm{HVA}}$ difference currents evoked by $2 \mathrm{nS}$ excitatory synaptic input to $D 3_{D}$ in models where the densities of $\mathrm{Ca}_{\mathrm{HVA}}$ and $S K$ have been dialed up or down in the compartments composing the $D 3_{D}$ stimulus site. Traces are aligned such that stimulus onsets are at zero. $D$, The negative region of $D 3_{D} P R C s$ is deeper for models where $\mathrm{Ca}_{\mathrm{HVA}}$ and $S K$ has been upregulated local to stimulus delivery. Elimination of either $\mathrm{Ca}_{\mathrm{HVA}}$ or $S K$ from D ${ }_{\mathrm{D}}$ yields visually identical type II PRCs, and like the $D 2_{D}$ case, the remaining negative regions of these PRCS (asterisk) stems from compartments adjacent to the stimulated region where the $\mathrm{Ca}_{\mathrm{HVA}} / \mathrm{SK}$ mechanism remains intact.

the conductance densities of $\mathrm{Ca}_{\mathrm{HVA}}$ and $\mathrm{SK}$ in the distal dendrite. Importantly, the intrinsic spiking rhythm of the model was not disturbed, because this manipulation was limited to the 25 distal dendritic compartments composing the stimulated site $\left(\mathrm{D} 2 \mathrm{D}_{\mathrm{D}}\right.$ or $\left.\mathrm{D} 3_{\mathrm{D}}\right)$. Local up- or downregulation of $\mathrm{Ca}_{\mathrm{HVA}}$ and $\mathrm{SK}$ in tandem directly scaled the magnitude of SK current evoked at the distal dendrite (Fig. 6A, C) and resulted in type II distal dendritic PRCs with deeper or shallower negative regions, respectively (Fig. $6 B, D)$. Removal of either $\mathrm{Ca}_{\mathrm{HVA}}$ or SK from $\mathrm{D} 2{ }_{\mathrm{D}}$ eliminated the type II character of the corresponding PRCs almost completely resulting in visually identical type I PRCs ( $r$ values of 0.0351 and 0.0357, respectively) (Fig. 6B; supplemental Fig. $6 C$, available at www.jneurosci.org as supplemental material). However, despite the local removal of the $\mathrm{Ca}_{\mathrm{HVA}}$ or SK conductance, these PRCs still contained shallow negative regions (Fig. $6 B$, asterisks) as a consequence of SK activation in compartments adjacent to the stimulated site. Activation of SK in compartments neighboring the $\mathrm{D} 3_{\mathrm{D}}$ stimulation site was particularly strong, and D $3_{\mathrm{D}}$ PRCs met our criterion for type II categorization even when $\mathrm{Ca}_{\mathrm{HVA}}$ or $\mathrm{SK}$ was removed from the site ( $r$ values of 0.78 and 0.80 , respectively) (Fig. 6D; supplemental Fig. $6 D$, available at www.jneurosci.org as supplemental material). Models with different distributions of SK conductance between the soma and dendrite (supplemental Fig. 3, available at www.jneurosci. org as supplemental material) also exhibited type II distal dendritic PRCs for which the negative region scaled in depth with the dendritic density of SK regardless of the somatic SK density (supplemental Fig. 7, available at www.jneurosci.org as supplemental material).

\section{Spike frequency dependence of somatic} and dendritic PRCs

Whereas GP neurons in slice typically spike spontaneously at frequencies below $15 \mathrm{~Hz}$, GP neurons in vivo spike with a mean frequency of $\sim 30 \mathrm{~Hz}$ in rodents (Ruskin et al., 1999; Urbain et al., 2000). A change in PRC shape with increasing firing frequency has been previously described for a simple $\theta$-neuron model and may shift synaptic integration from coincidence detection at low rates to acting as an integrator at high rates (Gutkin et al., 2005). To evaluate the dependence of PRC shape on spike frequency in our full morphological GP neuron model, we drove the model to $10-60 \mathrm{~Hz}$ firing frequency with 15-371 pA tonic somatic current injection.

During $7.9 \mathrm{~Hz}$ spontaneous spiking, only the ongoing ISI was affected by $2 \mathrm{nS}$ AMPA input to the soma. At this spike frequency, the PRC for the second interval (F2) was therefore flat (Figs. 7A3, 8A1) indicating the absence of higher-order effects of synaptic excitation.

During $30 \mathrm{~Hz}$ spiking, however, the soma was depolarized by $5 \mathrm{mV}$ on average compared with $10 \mathrm{~Hz}$ spiking (Fig. 7A1), and 2 nS AMPA inputs were capable of eliciting immediate spike initiation at earlier phases of the spike cycle than during $10 \mathrm{~Hz}$ spiking (Fig. 7A3,A4). This led to a left shift in the peak of the corresponding F1 PRCs (Fig. 8A1) for higher spike frequencies that was gradual between 10 and $60 \mathrm{~Hz}$. During $30 \mathrm{~Hz}$ spiking, the activation profile of somatic currents was substantially changed. Notably, when spiking was driven from $10 \mathrm{~Hz}$ to $30 \mathrm{~Hz}$, the baseline somatic SK current was elevated from $\sim 50 \mathrm{pA}$ to $\sim 125$ pA (Fig. 7A2) and stimulus elicited SK transients were additionally enhanced (Fig. 7A2, asterisk). As a consequence, during faster 


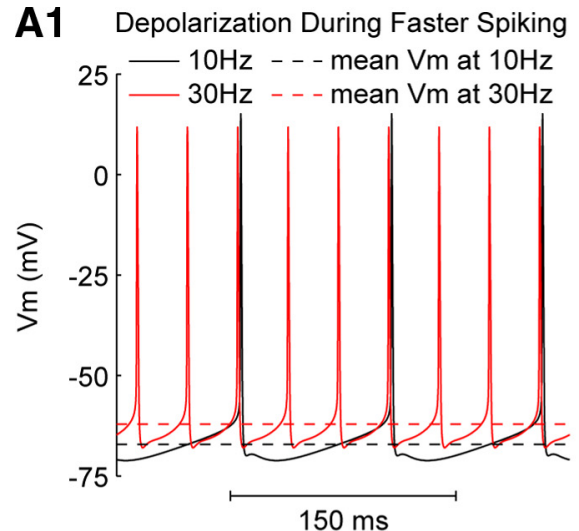

A2 Increased SK During Faster Spiking

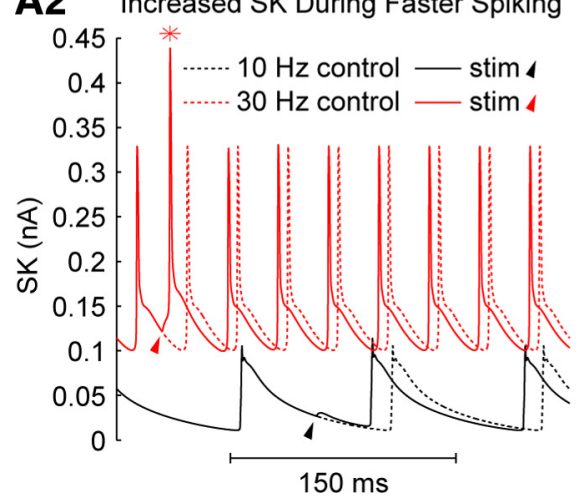

A3 Stimulated ISI \& 2nd Spike Cycle

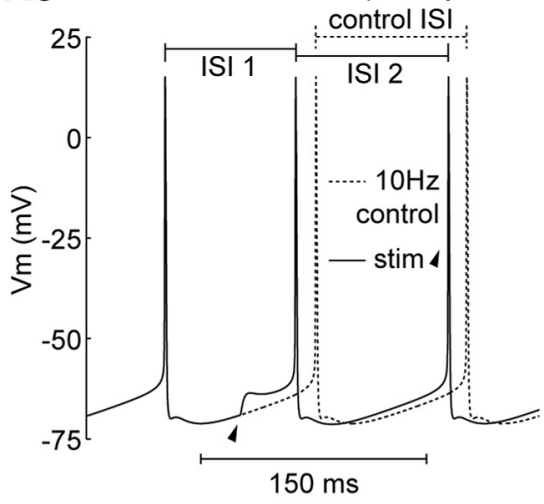

A4 Stimulated ISI \& 2nd Spike Cycle

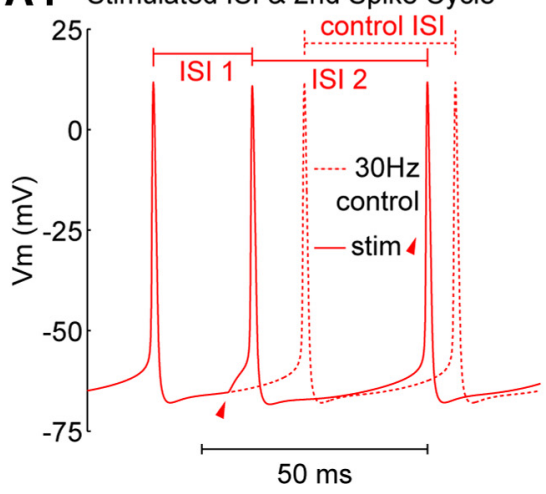

B1 D3D Stimulus-Evoked Voltage Deflection

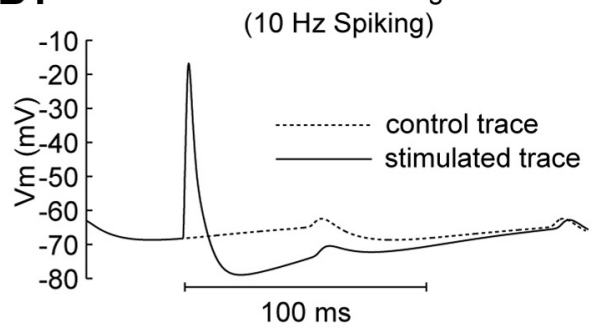

B2 D3D Stimulus-Evoked Voltage Deflection (30 Hz Spiking)

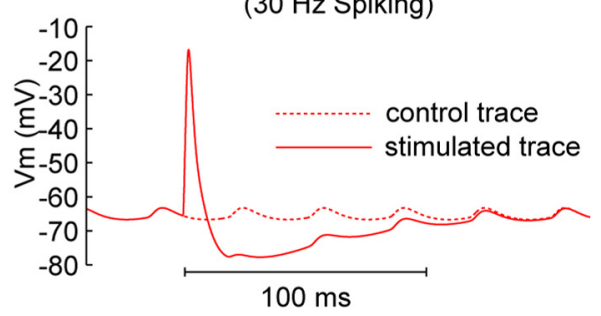

B3

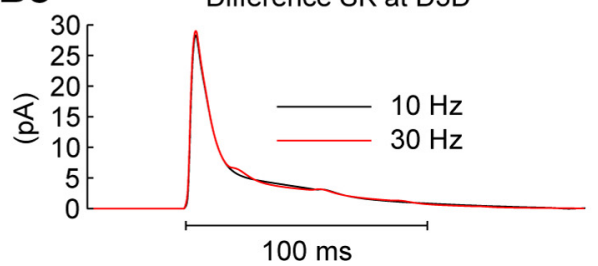

Figure 7. Spike frequency affects somatic but not distal dendritic SK activation. A, Faster spiking elevates baseline levels and augments stimulus-evoked transients of somatic SK current. $A \mathbf{A}$, When spiking was driven from $10 \mathrm{~Hz}$ (black trace) to $30 \mathrm{~Hz}$ (red trace) by increasing somatic applied current, the mean voltage at the soma (dashed black and red lines) was elevated $\sim 5 \mathrm{mV}$. A2, Driving faster spiking with somatic applied current elevated the baseline level of somatic SK current. Note that a $2 \mathrm{nS}$ AMPA stimulus (red and black arrowheads) elicited immediate initiation of a spike when applied to the soma during $30 \mathrm{~Hz}$ spiking but not when applied during $10 \mathrm{~Hz}$ spiking. The stimulus applied during $30 \mathrm{~Hz}$ spiking evoked a larger peak in SK current (red asterisk) than is evoked by spontaneous spikes (red dashed traces). A3. During $10 \mathrm{~Hz}$ spiking, the somatic stimulus (black arrowhead) shortened the ISI during which it was delivered, but did not affect the subsequent ISI. A4. During $30 \mathrm{~Hz}$ spiking the somatic stimulus (red arrowhead) shortened the ISI during which it was delivered. However, the subsequent ISI was Ionger in duration, reflecting the larger SK current evoked by the stimulus during faster spiking. $\boldsymbol{B}$, The time course of distal dendritic SK current flow is unaffected by spike frequency, spanning additional spike cycles during faster spiking. $\boldsymbol{B} 1$, During $10 \mathrm{~Hz}$ spiking, the local depolarization transient elicited by a $2 \mathrm{nS} \mathrm{AMPA} \mathrm{input} \mathrm{to} \mathrm{D3} 3_{\mathrm{D}}$ (solid black line) was $\sim 55 \mathrm{mV}$ relative to the control voltage trace (dashed black line). (Note that the ripples in the control voltage trace which demarcate the 100 ms spike cycles reflect attenuated spikes reaching the distal dendrite.) B2, During $30 \mathrm{~Hz}$ spiking, the local depolarization transient elicited by a $2 \mathrm{nS} A M P A$ input to $D 3_{D}$ (solid red line) was also $\sim 55 \mathrm{mV}$ relative to the control voltage trace (dashed red line). B3, The peak and time course of the SK difference current ( $~ 30 \mathrm{pA}$ ) evoked locally by AMPA inputs to D3 ${ }_{D}$ was not dependent on spike frequency. Difference current traces for $10 \mathrm{~Hz}$ (black) and $30 \mathrm{~Hz}$ (red) spiking overlay one another nearly perfectly.

spiking the somatic SK current activation accompanying spikes triggered by AMPA inputs was greater than the SK current activation accompanying control spikes. Therefore, the mAHP following an AMPA-evoked spike was slightly deeper than the mAHP during control spiking, and the subsequent spike cycle was longer than the control ISI (Fig. 7A4). While spike initiation immediately after AMPA stimulation corresponded to a large phase advance (F1; Fig. 8A1), a part of this advance was lost in the second spike cycle following stimulation (F2; Fig. 8A1), because this second ISI was longer than the baseline ISIs (Fig. 7A4). Therefore, at in vivo spike frequencies, an important effect of somatic SK conductance is to resist lasting advancements of the spiking rhythm. By lengthening the spike cycle following an AMPA-evoked spike, somatic SK makes the effect of the input more transient and significantly restores the original pattern of spiking. This effect of somatic SK conductance appears in the single-cycle somatic PRCs as a positive peak in the F1 that is nearly symmetrically opposed by a negative peak in the F2 (Fig. 8A1).

The so-called permanent PRC (Prinz et al., 2003a) consists of the sum of PRCs for the stimulated spike cycle and all subsequent spike cycles that show changed ISIs compared to baseline. Therefore, the permanent PRC reflects the lasting shift of the spike train (measured after several spike cycles) compared with the control oscillation. For somatic AMPA stimulation the permanent PRC remained type I for all spike frequencies from 10 to $60 \mathrm{~Hz}$ (Fig. $8 \mathrm{~A} 2$ ), indicating that the spike delay during the $\mathrm{F} 2$ period never exceeded the original spike advance in the stimulated spike cycle.

Distal dendritic voltage and current transients elicited by AMPA stimuli were generally unaffected by faster somatic spike rates (Fig. 7B1-B3), e.g., the $S K$ transient elicited by input to $D 3_{D}$ was identical at $10 \mathrm{~Hz}$ and $30 \mathrm{~Hz}$ firing frequencies (Fig. 7B3). Nevertheless, the PRC for $\mathrm{D} 3_{\mathrm{D}}$ stimulation showed a dramatic shift at faster somatic spike rates, because the elicited dendritic SK current had a fixed time course that now showed a different phase relationship with respect to the somatic spike cycle. The PRC of the stimulated spike cycle showed a positive peak shifted to the left, which resulted in a type I primary PRC for spike frequencies above $30 \mathrm{~Hz}$ (Fig. 8B1). Because the waveform of AMPA-evoked SK lasted $>50 \mathrm{~ms}$ (Fig. 7B3), at spike frequencies above $15 \mathrm{~Hz}$ the effect of SK current evoked by late-phase AMPA inputs to the distal dendrite impinged primarily on the second spike cycle (F2) following the stimulus. Consequently, the second ISI was prolonged, and the F2 PRC contained a significant negative peak for late-phase inputs (Fig. $8 \mathrm{B1}$ ). In the case of $\mathrm{D} 3_{\mathrm{D}}$ stimulation, the delayed spikes in $\mathrm{F} 2$ and $\mathrm{F} 3$ intervals more than compensated any 
A1
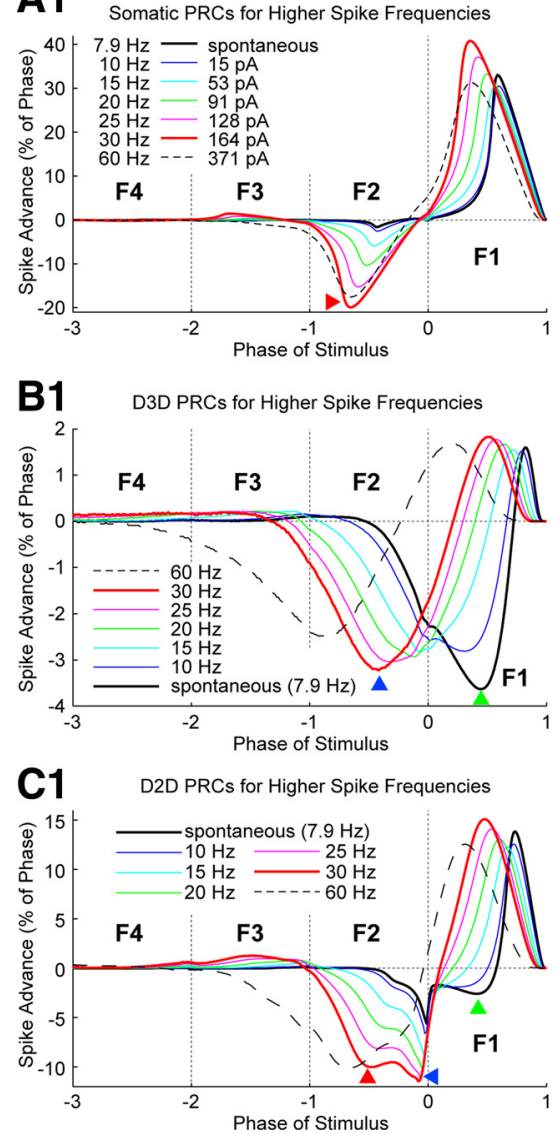

A2

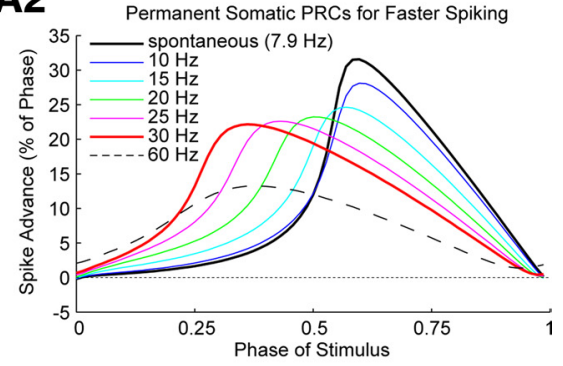

B2

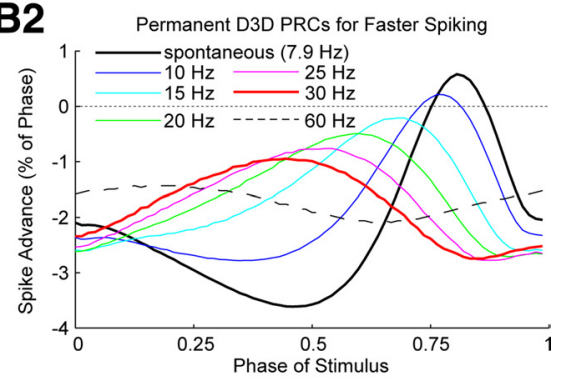

C2

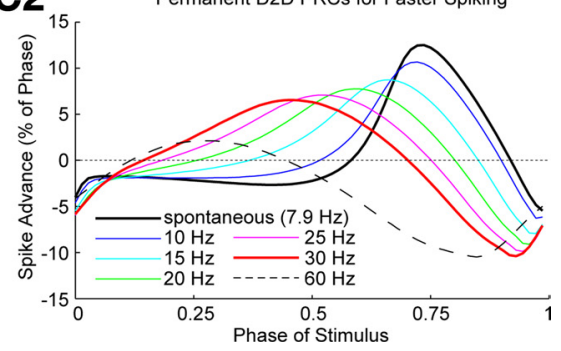

Figure 8. Faster axosomatic spiking changes the shapes of somatic and dendritic PRCs, and it is necessary to consider stimulus effects that outlast a single spike cycle. $\boldsymbol{A} 1$, When the model is driven faster (from $10 \mathrm{~Hz}$ to $30 \mathrm{~Hz}$ ) by increasing applied current to the soma, excitatory synaptic inputs of $2 \mathrm{nS}$ yield somatic PRCs with larger, earlier peaks and less skewness. $\boldsymbol{A 2}$, Permanent PRCs are calculated by summing single cycle PRCs F1 through F5. At higher spike frequencies the peak of the permanent somatic PRC is reduced, occurs earlier in phase, and is less skewed. $\mathbf{B}$, Like the somatic case, during faster spiking the positive peak of distal dendritic ( $\left(\mathrm{B}_{\mathrm{D}}\right)$ PRCS, occurs earlier in phase. The negative region present in $\mathrm{F} 1 \mathrm{PRCS}$ for $\mathrm{D} 3_{\mathrm{D}}$ inputs delivered during slower spiking (green arrowhead) is pushed into $\mathrm{F} 2$ when spiking is driven faster (blue arrowhead). $\mathbf{B 2}$, Permanent PRCs for D3 ${ }_{D}$ inputs have only a slight positive peak for the slowest spike frequencies, and at $15 \mathrm{~Hz}$ or faster, the entire permanent PRC is negative. C1, Single-cycle $P R C S$ for inputs to $D 2_{D}$ show an amalgam of the effects of somatic or $D 3_{D}$ stimulation. The red and blue arrowheads correspond to the red arrowhead in $A 1$ and the blue arrowhead in $B 1$. C2, The permanent PRCS for $D 2_{D}$ inputs are type II for all spike frequencies up to $60 \mathrm{~Hz}$, and they vary smoothly as a function of spike frequency. For high spike frequencies a negative region develops for stimuli late in phase.

spike advance occurring in the stimulated spike cycle. The corresponding permanent PRC became entirely negative at spike frequencies above $10 \mathrm{~Hz}$, i.e., a strong AMPA stimulus at any phase of the spike cycle ultimately resulted in a delay of the spike oscillation. Therefore, our model predicts that at in vivo spike rates strong AMPA input to the distal dendrites of GP neurons will advance the first subsequent spike but ultimately reduce spike frequency.

Stimulus locations at intermediate electrotonic distance from the soma showed a combination of the effects seen at the soma and the distal dendrite. The voltage at these locations was somewhat more depolarized during faster spiking (as at the soma), but local SK transients evoked by AMPA inputs were still relatively invariant across spike frequencies (as at the more-distal dendrite). Like the $\mathrm{D} 3_{\mathrm{D}}$ case, AMPA stimuli applied late in the spike cycle led to a negative region in the PRC that moved from the F1 to the F2 ISI for increasing spike rates (Fig. $8 \mathrm{~B} 1, \mathrm{C1}$, blue arrowheads). The lasting effects of AMPA inputs on spike timing were similar for $\mathrm{D} 2_{\mathrm{D}}$ and $\mathrm{D} 3_{\mathrm{D}}$, such that the peak of the permanent
PRC was shifted leftward and became flatter at higher spike frequencies (Fig. 8 B2,C2). However, for mid-distal AMPA stimuli the PRC retained a positive region for all spike frequencies, and thus remained type II.

It will be of significant interest to evaluate how well the permanent PRC explains the emergence of synchronous states of network activity, or whether the development of network synchrony from asynchronous modes is better explained by the single-cycle primary and higherorder PRCs. It is important to note that the time-period during which the SK current evoked by excitatory dendritic stimulation is strongest lasts $\sim 40 \mathrm{~ms}(\sim 25$ $\mathrm{Hz}$ ), suggesting the possibility that dendritic SK current could cause GP neurons to resonate with $\beta$-frequency oscillatory excitation from STN.

\section{Dendritic conductance densities modulate dendritic PRCs}

The preponderance of synaptic inputs on GP neurons is located on dendrites, which are quite long, but thin and sparsely branching (Falls et al., 1983; Shink and Smith, 1995). To understand how dendritic inputs remote from the soma could influence spiking one needs to consider the local processing of inputs by active conductances in the dendrite. However, dendritic currents have not been recorded experimentally because the dendritic diameter is generally too thin for whole-cell recording. Therefore dendritic conductance properties are not well constrained for GP neurons and the assumptions of our model with respect to dendritic conductance densities are tentative, and predominantly derived from matching somatic current-clamp data. To examine the robustness of our findings with respect to parameter variations of dendritic conductance densities, we varied dendritic conductance densities over a large range and determined the consequences for PRCs. Specifically, we either adjusted the fast spike conductances $\left(\mathrm{NaF}, \mathrm{NaP}, \mathrm{K}_{\mathrm{V}} 3\right.$, and $\left.\mathrm{K}_{\mathrm{V}} 2\right)$ together between 0 and $150 \%$ of their baseline values, or we separately adjusted the major slow dendritic inward $(\mathrm{NaP})$ or outward (SK) currents.

We focus our analysis of how manipulating dendritic parameters affects PRCs on $2.5 \mathrm{nS}$ AMPA stimulation of the $\mathrm{D} 2_{\mathrm{D}}$ stimulation site, because this location showed a combination of local dendritic and somatodendritic coupling effects in our preceding analyses (Fig. 8). To eliminate spike rate effects on PRC shape, we drove all models to $30 \mathrm{~Hz}$ spiking with somatic current injection to match in vivo rates (103-202 pA current depending on dendritic parameters). Varying the density of $\mathrm{NaP}$ alone or of the 4 spike conductances together had relatively slight effects on PRC shape that were similar between the two manipulations. Notably, for the largest values of $\mathrm{NaP}$ or the spike currents both the maximal spike advances in the stimulated spike cycle (F1) and the 
maximal spike delays in the subsequent spike cycle (F2) were increased (Fig. $9 A 1, B 1)$. The combination of these effects led to permanent PRCs that showed an increased spike advance for AMPA stimulation at mid spike-cycle and increased spike delay for AMPA stimulation at the beginning or end of the spike cycle (Fig. $9 A 2, B 2)$. These effects can be understood by examining the differences in dendritic membrane currents between models with different dendritic conductance densities (supplemental Fig. 8, available at www. jneurosci.org as supplemental material). Higher dendritic densities of $\mathrm{NaP}$ or of the spike conductances increased the positive peaks of the corresponding F1 PRCs by supporting larger inward current transients, and increased the negative regions of the corresponding F2 PRCs by amplifying outward evoked SK currents. Consequently the permanent PRCs showed increased positive and negative peaks while the basic shape was retained (Fig. 9A2). Overall, these manipulations show that the PRC results we have described are quite robust even for large changes in the density of persistent inward and spike currents. The dependencies of PRC shape on dendritic conductance densities also provide insight into important potential sources of heterogeneity in pallidal networks, and they illustrate potential mechanisms of modulatory influence on neuronal and network dynamics. Note that we kept the increase in spike currents below the threshold for full blown propagating dendritic action potentials. When spike conductance densities were sufficient to support dendritic spike initiation in response to AMPA input, the type II character of dendritic PRCs disappeared and spike advances were much more pronounced (supplemental Fig. 9, available at www.jneurosci.org as supplemental material). Thus, neurons or neuron models with spiking dendrites should be seen as integrating dendritic inputs in a completely different manner resulting in type I PRCs.

\section{Dendritic SK conductance}

As described above, we found that dendritic SK can powerfully alter PRC shape by converting the inward synaptic current of an AMPA input into a net outward membrane charge flow and resulting in spike delays for early-phase inputs. Not surprisingly, decreasing or increasing the dendritic SK density had important effects on dendritic PRCs (Fig. 9C). When SK conductance was removed from the dendrite, the permanent PRC became entirely positive (Fig. 9C2, red trace). In this case, activation of somatic SK still resulted in F2 spike delays, because the advancement of the first (F1) spike led to a larger somatic SK current that impacted the second (F2) spike cycle (Fig. 9C1, blue arrowhead). Increasing the density of dendritic SK smoothly affected the corresponding PRCs. Models with greater dendritic SK showed a diminished positive peak in the F1 PRC and a larger negative peak in the F2
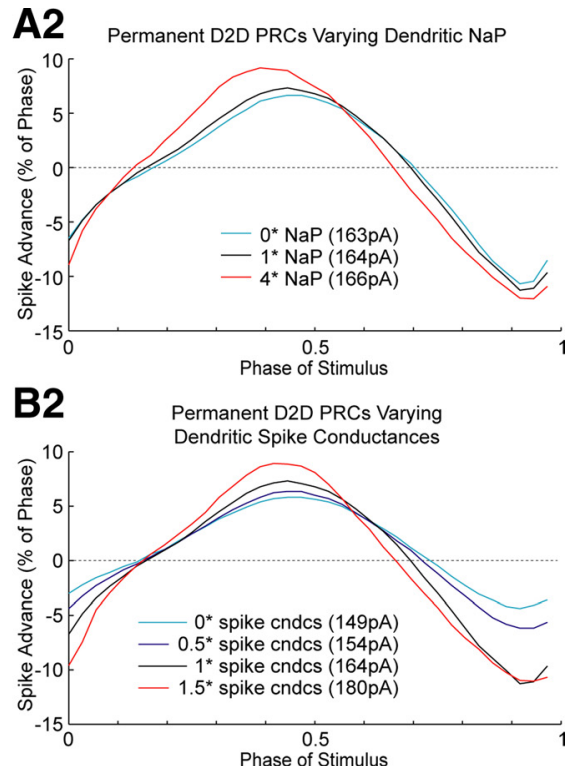

C2 Permanent D2D PRCs Varying Dendritic SK

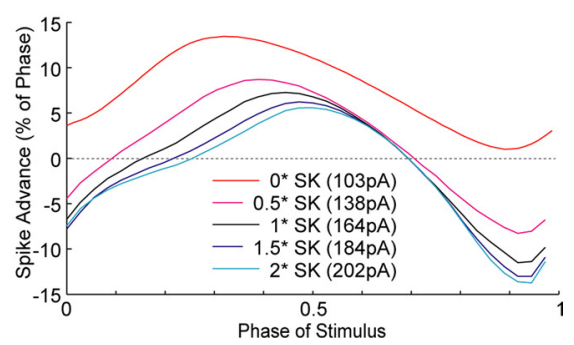

Phase of Stimulus

Figure 9. Distal dendritic PRCs are robustly type II against significant variation of dendritic conductance densities. $\boldsymbol{A}$, Singlecycle PRCs (A1, F1-F4) and the permanent PRC ( $\boldsymbol{A} 2)$ for models with 0, 1, and 4 times the base dendritic density of NaP. For models with greater dendritic densities of NaP the positive peak in the F1 PRC and both of the negative peaks in the F2 PRC were increased. 列 densities of $S K$, the positive peak in the F1 D2 PRC and the early-phase negative peak (blue arrowhead) is reduced, whereas the late-phase negative peak in the $\mathrm{F} 2 \mathrm{D} 2{ }_{\mathrm{D}} \mathrm{PRC}$ (solid red arrowhead) is increased. Complete removal of SK conductance from the dendrite (red traces in $\mathbf{C}$ and $\mathbf{C 2}$ ) eliminates the late-phase negative peak in the F2 D2 $2_{D}$ PRC (red box arrowhead) and the corresponding permanent PRC is purely positive.

PRC (Fig. 9C1, red arrowhead). The mechanism underlying this result is straightforward, as increased dendritic SK conductance led to increased outward current and greater spike delays in response to dendritic AMPA inputs (supplemental Fig. 8, available at www.jneurosci.org as supplemental material). Other effects of increasing dendritic SK were mild, mainly resulting in slight hyperpolarization of the dendrite during the control spike cycle and a concomitant decrease of other depolarization-activated currents. These results indicate that dendritic SK can be varied over a large range while maintaining a smoothly changing type II dendritic PRC, whereas a complete absence of dendritic SK would lead to type I phase response dynamics.

\section{Type II dendritic PRCs are robust within a large range of SK activation parameters}

There is uncertainty in our model about kinetic parameter settings governing SK activation, because the precise calcium dynamics governing SK activation as well as SK activation time constants in GP neurons are not experimentally well constrained. Therefore, we performed a final set of simulation experiments to determine the dependence of our results on variables central to 
the mechanism of SK activation that could importantly affect phase response dynamics in response to dendritic excitation. SK was activated by inward calcium pulses carried by the $\mathrm{Ca}_{\mathrm{HVA}}$ conductance. The transient spike in intracellular calcium elicited by an AMPA input to the dendrite lasted $<10 \mathrm{~ms}$ in our simulations (supplemental Fig. 10, available at www.jneurosci.org as supplemental material), approximating the dynamics of the interaction of calcium channels with SK channels in membrane micro-domains. However, SK deactivated relatively slowly as a consequence of the default SK deactivation time constant of 76 $\mathrm{ms}$ in our simulations. This slow deactivation of SK in the model prolonged SK current transients that shaped the spontaneous mAHP to match experimental data (Günay et al., 2008). Direct measurements of SK deactivation in oocyte expression systems indicate time constants between 22 and 38 ms (Xia et al., 1998), consistent with a relatively slow calcium dependent deactivation.

To determine the effects of key kinetic parameters affecting SK activation on PRC behavior we varied (1) the rate of intracellular calcium clearance between 0.25 and $2 \mathrm{~ms}$, (2) the steady-state calcium dependence of SK activation between 0.175 and $1 \mu \mathrm{M}$, and (3) the time constant of calcium-dependent SK activation between 25 and $100 \mathrm{~ms}$ (supplemental Fig. 11, available at www. jneurosci.org as supplemental material). Each of these manipulations affected the spontaneous spike frequency, so each model was again driven to $30 \mathrm{~Hz}$ by somatic applied current for PRC analysis. Permanent D2 ${ }_{D}$ PRCs were type II for all levels of each manipulation, although the amplitude and skewness were modulated by varying the activation and kinetic parameters for SK. Therefore, the dendritic mechanism that yields type II dynamics in our model was not a consequence of specific parameter choices and is likely to encompass the regime of dendritic processing of strong AMPA inputs in vivo.

\section{Discussion}

The primary result of our analysis of phase response curves of GP model neurons was that perisomatic and dendritic excitatory inputs yielded distinctly different PRCs. Perisomatic excitation at all times in the spike cycle led to spike advances and thus a type I PRC, whereas dendritic excitation led to spike delays for inputs early in the spike cycle thus yielding a biphasic type II PRC. We found that the type II character of dendritic PRCs was the consequence of stimulus-elicited calcium transients and subsequent SK activation that occurred even for stimuli simulating a single small distal synaptic input. Type II PRCs can enhance the ability of neurons to synchronize in connected networks (Hansel et al., 1995; Goel and Ermentrout, 2002; Galán et al., 2007a; Tsubo et al., 2007; Bogaard et al., 2009) and populations of neurons with type II PRCs synchronize more readily to correlated inputs (Galán et al., 2007b; Marella and Ermentrout, 2008; Abouzeid and Ermentrout, 2009). Because most excitatory input to GP neurons is on dendritic sites (Shink and Smith, 1995) our results suggests a novel cellular mechanism important in the integration of excitatory input in GP network activity in normal conditions and to the pathological synchronization of GP neurons in Parkinson's disease.

\section{Phase response curve analysis of a morphological GP neuron model}

We turned to computer modeling to examine detailed PRC behavior in GP neurons, as spike firing even in brain slices is not sufficiently regular or precise (Deister et al., 2009) to reliably quantify small spike shifts caused by individual synaptic inputs.
In addition application of dendritic inputs of controlled amplitude and location is not currently experimentally feasible. These factors put severe limits on the experimental measurement of PRCs.

\section{GP model construction}

For this study we adapted a GP neuron model that we have previously used to investigate the influence of conductance densities on spiking properties (Günay et al., 2008). We observed that most electrophysiological properties of the models were affected by multiple conductances, and each conductance affected multiple electrophysiological measures (Günay et al., 2008), which is consistent with modeling studies of other types of neurons (Prinz et al., 2003b; Achard and De Schutter, 2006; Taylor et al., 2009). Adaptations to the model were primarily made to include new experimental findings on sodium channel properties in GP neurons (Mercer et al., 2007). The new base model was located in a broad basin of models with similar spiking properties when channel densities were varied, as described in our earlier publication (Günay et al., 2008). Hence, it is important to note that the model used here does not represent a unique solution to GP spiking properties, but that all findings are robust against considerable parameter variations.

\section{Dendritic properties}

The architecture of dendritic trees and the complement of active dendritic conductances are critical features of the computational machinery used by neurons with diverse functional objectives (Mainen and Sejnowski, 1996; Vetter et al., 2001; Krichmar et al., 2002; Stiefel and Sejnowski, 2007; Chen et al., 2009; Komendantov and Ascoli, 2009). We used full morphological reconstructions from biocytin filled neurons to obtain a correct representation of the thin, long and sparsely branched structure of GP dendrites (Falls et al., 1983). No dendritic recordings of GP dendrites exist, leading to considerable uncertainty about dendritic current densities in GP. Channel antibody staining has revealed the presence of voltage-gated sodium channels (Hanson et al., 2004) and calcium channels (Hanson and Smith, 2002) in GP dendrites, however. Somatic conductances in GP have been characterized in multiple studies (Baranauskas et al., 1999, 2003; Tkatch et al., 2000; Chan et al., 2004; Mercer et al., 2007) and were matched to these results in our model (Günay et al., 2008). The SK conductance has been found to govern the mAHP waveform in GP neurons (Deister et al., 2009) and in other cell types has been shown to be activated by calcium inflow through L-type (Marrion and Tavalin, 1998) or N-type (Hallworth et al., 2003; Goldberg and Wilson, 2005) HVA calcium channels. Immunocytochemistry showed an increased density of L- and N-type HVA calcium channels in GP dendrites (Hanson and Smith, 2002), which would support a high level of dendritic SK activation. Dendritic SK has not been examined in GP, but has been found to be present in other types of neurons with SK conductance (Sailer et al., 2002; Cai et al., 2004; Maher and Westbrook, 2005).

\section{Dependence of PRC shape on physiological properties}

Traditional PRC analysis relies on linear scaling of PRCs for positive and negative stimuli of varying amplitudes. We found that excitatory dendritic inputs in particular may not have a regime of weak coupling in GP, because the local input resistance is so high that any stimulus of physiological amplitude results in local depolarization that triggers changes in active conductances and hence response nonlinearities. Dendritic depolarization following AMPA inputs leads to a transformation of a type I infinitesimal PRC for very small $(<1 \mathrm{pA})$ stimuli to a type II PRC for 
physiologically sized stimuli due to SK activation. The property of symmetry for positive and negative stimuli was also lost, as GABA inputs were associated with type I PRCs at all amplitudes. These results were robust for a large range of $\mathrm{Ca}_{\mathrm{HVA}}$ and $\mathrm{SK}$ conductance densities and different parameters for SK activation kinetics, though the precise shape of biphasic PRCs smoothly changed when parameters were varied. Another important observation was that when spike frequency was driven to frequencies typically observed in vivo, the negative region of biphasic PRCs was pushed into the F2 spike interval, because the time course of SK activation with AMPA stimulation exceeded the interspike interval at fast firing frequencies.

\section{Implications of PRC behavior for GP network interactions}

To make PRC analysis analytically tractable, it is most commonly implemented in silico using brief, weak perturbations applied to simple point neuron models that lack a full contingent of membrane conductances. In such cases explicit predictions of phaselocked modes can be made for networks with well defined connectivity architectures, and type II PRCs have been associated with synchronization in networks coupled through excitation (Hansel et al., 1995; Goel and Ermentrout, 2002; Galán et al., 2007a; Tsubo et al., 2007). Further work recognized the importance of active conductances in shaping PRC behavior. Ermentrout et al. (2001) and Gutkin et al. (2005) evaluated the contribution of a spike frequency adaptation (SFA) potassium current to skewness of PRCs and the consequent emergence of network synchronization. Recently, Stiefel and colleagues $(2008,2009)$ demonstrated that in principle cholinergic modulation of cortical pyramidal neurons could switch the PRC from type II to type I as a consequence of SFA current $\left(I_{\mathrm{M}}\right)$ down regulation.

Dendrites can change PRC shape through an attenuation and delay of an input's effect on somatic spiking and hence a left-shift of the dendritic PRC in the domain of weak coupling (Goldberg et al., 2007). It has also been shown that the electrotonic length of dendritic cables can determine the mode of synchronization in a reciprocal network of two distally coupled ball-and-stick neuron models (Crook et al., 1998a). These studies demonstrate that active dendritic conductances can sculpt effective input waveforms and compensate for the passive filtering properties of dendrites. Our own results indicate that active dendritic conductances can have far reaching consequences for dendritic PRCs, and that SK conductance in particular can lead to dendritic type II PRC behavior in neurons showing somatic type I PRCs. Thus, dendritic conductances can bestow phase response characteristics that are not predicted by somatic excitability, causing the dendrite to act as a distinct dynamical subsystem. This mechanism also gives excitatory input a special relevance for network synchronization, as type II phase response properties were not observed with inhibition.

Given that phenomena of network synchronization are highly sensitive to PRC shapes, our finding of type II dendritic PRCs in GP likely has important consequences for considering network activity in globus pallidus. GP neurons shift from an asynchronous firing mode to synchronous bursting in PD that is phaselocked to oscillations in other basal ganglia nuclei (Bergman et al., 1994; Nini et al., 1995; Raz et al., 2000). An important mechanism underlying synchronization in basal ganglia networks is believed to be given by STN-GP feedback activity (Plenz and Kitai, 1999; Bevan et al., 2002; Terman et al., 2002). For cortical slow-wave oscillations elicited under anesthesia, STN fires bursts in synchrony with cortical oscillations in normal or dopamine depleted animals, whereas GP only shows robust synchronized bursting following dopamine depletion (Magill et al., 2001). This result suggests that modulation in the parkinsonian state may induce a change in the propensity of GP neurons to engage in network synchronization. Synchronization of pallidal networks would in turn increase the effectiveness of GP inhibition to cause correlated pauses and rebound bursting in STN (Terman et al., 2002), and entrainment of GP neurons to $\beta$-frequency STN oscillations would promote resonance between these coupled nuclei. Previous work suggested that HCN channels play a major role in GP neurons in controlling firing regularity and bursting in PD (Chan et al., 2004). Our PRC analysis suggests that in addition dendritic SK may be a key determinant in controlling synchronization through changing the phase dependence of synaptic effects on spike timing. Dopamine modulates SK conductance through $\mathrm{Ca}_{\mathrm{V}} 2.2$ downregulation in STN neurons (Ramanathan et al., 2008). If this mechanism holds for GP neurons as well, $\mathrm{a} \mathrm{Ca}_{\mathrm{V}} 2.2$ upregulation and hence increased SK activation and an increase in type II phase response dynamics might be expected in PD based on our results.

\section{References}

Abouzeid A, Ermentrout B (2009) Type-II phase resetting curve is optimal for stochastic synchrony. Phys Rev E Stat Nonlin Soft Matter Phys 80:011911.

Achard P, De Schutter E (2006) Complex parameter landscape for a complex neuron model. PLoS Comput Biol 2:e94.

Achuthan S, Canavier CC (2009) Phase-resetting curves determine synchronization, phase locking, and clustering in networks of neural oscillators. J Neurosci 29:5218-5233.

Acker CD, Kopell N, White JA (2003) Synchronization of strongly coupled excitatory neurons: relating network behavior to biophysics. J Comput Neurosci 15:71-90.

Baranauskas G, Tkatch T, Surmeier DJ (1999) Delayed rectifier currents in rat globus pallidus neurons are attributable to Kv2.1 and Kv3.1/3.2 K+ channels. J Neurosci 19:6394-6404.

Baranauskas G, Tkatch T, Nagata K, Yeh JZ, Surmeier DJ (2003) Kv3.4 subunits enhance the repolarizing efficiency of Kv3.1 channels in fast-spiking neurons. Nat Neurosci 6:258-266.

Bergman H, Wichmann T, Karmon B, DeLong MR (1994) The primate subthalamic nucleus. 2. Neuronal-activity in the Mptp model of Parkinsonism. J Neurophysiol 72:507-520.

Bevan MD, Magill PJ, Terman D, Bolam JP, Wilson CJ (2002) Move to the rhythm: oscillations in the subthalamic nucleus-external globus pallidus network. Trends Neurosci 25:525-531.

Bogaard A, Parent J, Zochowski M, Booth V (2009) Interaction of cellular and network mechanisms in spatiotemporal pattern formation in neuronal networks. J Neurosci 29:1677-1687.

Cai X, Liang CW, Muralidharan S, Kao JP, Tang CM, Thompson SM (2004) Unique roles of SK and Kv4.2 potassium channels in dendritic integration. Neuron 44:351-364.

Chan CS, Shigemoto R, Mercer JN, Surmeier DJ (2004) HCN2 and HCN1 channels govern the regularity of autonomous pacemaking and synaptic resetting in globus pallidus neurons. J Neurosci 24:9921-9932.

Chen CC, Abrams S, Pinhas A, Brumberg JC (2009) Morphological heterogeneity of layer VI neurons in mouse barrel cortex. J Comp Neurol 512:726-746.

Crook SM, Ermentrout GB, Bower JM (1998a) Dendritic and synaptic effects in systems of coupled cortical oscillators. J Comput Neurosci 5:315-329.

Crook SM, Ermentrout GB, Bower JM (1998b) Spike frequency adaptation affects the synchronization properties of networks of cortical oscillators. Neural Comput 10:837-854.

Deister CA, Wilson CJ (2008) Variability of fast afterhyperpolarization currents reduces the regularity of spontaneous firing in globus pallidus neurons. Soc Neurosci Abstr 34:43.17.

Deister CA, Chan CS, Surmeier DJ, Wilson CJ (2009) Calcium-activated SK channels influence voltage-gated ion channels to determine the precision of firing in globus pallidus neurons. J Neurosci 29:8452-8461.

Ermentrout B (1996) Type I membranes, phase resetting curves, and synchrony. Neural Comput 8:979-1001.

Ermentrout B, Pascal M, Gutkin B (2001) The effects of spike frequency 
adaptation and negative feedback on the synchronization of neural oscillators. Neural Comput 13:1285-1310.

Falls WM, Park MR, Kitai ST (1983) An intracellular HRP study of the rat globus pallidus. II. Fine structural characteristics and synaptic connections of medially located large GP neurons. J Comp Neurol 221:229-245.

Galán RF, Ermentrout GB, Urban NN (2007a) Reliability and stochastic synchronization in type I vs. type II neural oscillators. Neurocomputing 70:2102-2106.

Galán RF, Ermentrout GB, Urban NN (2007b) Stochastic dynamics of uncoupled neural oscillators: Fokker-Planck studies with the finite element method. Phys Rev E Stat Nonlin Soft Matter Phys 76:06110.

Gamper N, Stockand JD, Shapiro MS (2003) Subunit-specific modulation of KCNQ potassium channels by Src tyrosine kinase. J Neurosci 23:84-95.

Goel P, Ermentrout B (2002) Synchrony, stability, and firing patterns in pulse-coupled oscillators. Physica D 163:191-216.

Goldberg JA, Wilson CJ (2005) Control of spontaneous firing patterns by the selective coupling of calcium currents to calcium-activated potassium currents in striatal cholinergic interneurons. J Neurosci 25:10230-10238.

Goldberg JA, Deister CA, Wilson CJ (2007) Response properties and synchronization of rhythmically firing dendritic neurons. J Neurophysiol 97:208-219.

Günay C, Edgerton JR, Jaeger D (2008) Channel density distributions explain spiking variability in the globus pallidus: a combined physiology and computer simulation database approach. J Neurosci 28:7476-7491.

Gutkin BS, Ermentrout GB, Reyes AD (2005) Phase-response curves give the responses of neurons to transient inputs. J Neurophysiol 94:1623-1635.

Hallworth NE, Wilson CJ, Bevan MD (2003) Apamin-sensitive small conductance calcium-activated potassium channels, through their selective coupling to voltage-gated calcium channels, are critical determinants of the precision, pace, and pattern of action potential generation in rat subthalamic nucleus neurons in vitro. J Neurosci 23:7525-7542.

Hansel D, Mato G, Meunier C (1995) Synchrony in excitatory neural networks. Neural Comput 7:307-337.

Hanson JE, Smith Y (2002) Subcellular distribution of high-voltageactivated calcium channel subtypes in rat globus pallidus neurons. J Comp Neurol 442:89-98.

Hanson JE, Smith Y, Jaeger D (2004) Sodium channels and dendritic spike initiation at excitatory synapses in globus pallidus neurons. J Neurosci 24:329-340.

Hernández A, Ibáñez-Sandoval O, Sierra A, Valdiosera R, Tapia D, Anaya V, Galarraga E, Bargas J, Aceves J (2006) Control of the subthalamic innervation of the rat globus pallidus by $\mathrm{D}-2 / 3$ and $\mathrm{D}-4$ dopamine receptors. J Neurophysiol 96:2877-2888.

Hirschberg B, Maylie J, Adelman JP, Marrion NV (1998) Gating of recombinant small-conductance Ca-activated $\mathrm{K}+$ channels by calcium. J Gen Physiol 111:565-581.

Hirschberg B, Maylie J, Adelman JP, Marrion NV (1999) Gating properties of single SK channels in hippocampal CA1 pyramidal neurons. Biophys J 77:1905-1913.

Keen JE, Khawaled R, Farrens DL, Neelands T, Rivard A, Bond CT, Janowsky A, Fakler B, Adelman JP, Maylie J (1999) Domains responsible for constitutive and $\mathrm{Ca}^{2+}$-dependent interactions between calmodulin and small conductance $\mathrm{Ca}^{2+}$ activated potassium channels. J Neurosci 19:8830-8838.

Khaliq ZM, Gouwens NW, Raman IM (2003) The contribution of resurgent sodium current to high-frequency firing in Purkinje neurons: an experimental and modeling study. J Neurosci 23:4899-4912.

Kita H, Kitai ST (1994) The morphology of globus pallidus projection neurons in the rat: an intracellular staining study. Brain Res 636:308-319.

Komendantov AO, Ascoli GA (2009) Dendritic excitability and neuronal morphology as determinants of synaptic efficacy. J Neurophysiol 101:1847-1866.

Krichmar JL, Nasuto SJ, Scorcioni R, Washington SD, Ascoli GA (2002) Effects of dendritic morphology on CA3 pyramidal cell electrophysiology: a simulation study. Brain Res 941:11-28.

Loucif KC, Wilson CL, Baig R, Lacey MG, Stanford IM (2005) Functional interconnectivity between the globus pallidus and the subthalamic nucleus in the mouse brain slice. J Physiol 567:977-987.

Magill PJ, Bolam JP, Bevan MD (2000) Relationship of activity in the sub- thalamic nucleus-globus pallidus network to cortical electroencephalogram. J Neurosci 20:820-833.

Magill PJ, Bolam JP, Bevan MD (2001) Dopamine regulates the impact of the cerebral cortex on the subthalamic nucleus-globus pallidus network. Neuroscience 106:313-330.

Magistretti J, Alonso A (1999) Biophysical properties and slow voltagedependent inactivation of a sustained sodium current in entorhinal cortex layer-II principal neurons-a whole-cell and single-channel study. J Gen Physiol 114:491-509.

Magistretti J, Alonso A (2002) Fine gating properties of channels responsible for persistent sodium current generation in entorhinal cortex neurons. J Gen Physiol 120:855-873.

Magistretti J, Ragsdale DS, Alonso A (1999) Direct demonstration of persistent $\mathrm{Na}+$ channel activity in dendritic processes of mammalian cortical neurones. J Physiol 521:629-636.

Maher BJ, Westbrook GL (2005) SK channel regulation of dendritic excitability and dendrodendritic inhibition in the olfactory bulb. J Neurophysiol 94:3743-3750.

Mainen ZF, Sejnowski TJ (1996) Influence of dendritic structure on firing pattern in model neocortical neurons. Nature 382:363-366.

Mallet N, Pogosyan A, Márton LF, Bolam JP, Brown P, Magill PJ (2008) Parkinsonian beta oscillations in the external globus pallidus and their relationship with subthalamic nucleus activity. J Neurosci 28:14245-14258.

Marella S, Ermentrout GB (2008) Class-II neurons display a higher degree of stochastic synchronization than class-I neurons. Phys Rev E Stat Nonlin Soft Matter Phys 77:041918.

Marrion NV, Tavalin SJ (1998) Selective activation of Ca2+-activated K+ channels by co-localized Ca2 + channels in hippocampal neurons. Nature 395:900-905.

Mercer JN, Chan CS, Tkatch T, Held J, Surmeier DJ (2007) Nav1.6 sodium channels are critical to pacemaking and fast spiking in globus pallidus neurons. J Neurosci 27:13552-13566.

Netoff TI, Clewley R, Arno S, Keck T, White JA (2004) Epilepsy in smallworld networks. J Neurosci 24:8075-8083.

Netoff TI, Acker CD, Bettencourt JC, White JA (2005a) Beyond two-cell networks: Experimental measurement of neuronal responses to multiple synaptic inputs. J Comput Neurosci 18:287-295.

Netoff TI, Banks MI, Dorval AD, Acker CD, Haas JS, Kopell N, White JA (2005b) Synchronization in hybrid neuronal networks of the hippocampal formation. J Neurophysiol 93:1197-1208.

Nini A, Feingold A, Slovin H, Bergman H (1995) Neurons in the globus pallidus do not show correlated activity in the normal monkey, but phaselocked oscillations appear in the MPTP model of parkinsonism. J Neurophysiol 74:1800-1805.

Oprisan SA, Canavier CC (2002) The influence of limit cycle topology on the phase resetting curve. Neural Comput 14:1027-1057.

Park MR, Falls WM, Kitai ST (1982) An intracellular HRP study of the rat globus pallidus. I. Responses and light microscopic analysis. J Comp Neurol 211:284-294.

Plenz D, Kital ST (1999) A basal ganglia pacemaker formed by the subthalamic nucleus and external globus pallidus. Nature 400:677-682.

Preyer AJ, Butera RJ (2005) Neuronal oscillators in Aplysia californica that demonstrate weak coupling in vitro. Phys Rev Lett 95:138103.

Prinz AA, Thirumalai V, Marder E (2003a) The functional consequences of changes in the strength and duration of synaptic inputs to oscillatory neurons. J Neurosci 23:943-954.

Prinz AA, Billimoria CP, Marder E (2003b) Alternative to hand-tuning conductance-based models: construction and analysis of databases of model neurons. J Neurophysiol 90:3998-4015.

Prole DL, Marrion NV (2004) Ionic permeation and conduction properties of neuronal KCNQ2/KCNQ3 potassium channels. Biophys J $86: 1454-1469$.

Raman IM, Bean BP (2001) Inactivation and recovery of sodium currents in cerebellar Purkinje neurons: evidence for two mechanisms. Biophys J 80:729-737.

Ramanathan S, Tkatch T, Atherton JF, Wilson CJ, Bevan MD (2008) D-2like dopamine receptors modulate SKCa channel function in subthalamic nucleus neurons through inhibition of $\mathrm{Ca}(\mathrm{v}) 2.2$ channels. J Neurophysiol 99:442-459.

Raz A, Vaadia E, Bergman H (2000) Firing patterns and correlations of spontaneous discharge of pallidal neurons in the normal and the tremu- 
lous 1-methyl-4-phenyl-1,2,3,6-tetrahydropyridine vervet model of parkinsonism. J Neurosci 20:8559-8571.

Rinzel JM, Ermentrout GB (1998) Analysis of neural excitability and oscillations. In: Methods in neuronal modeling, Ed 2 (Koch C, Segev I, eds), pp 251-291. Cambridge, MA: MIT Press.

Ruskin DN, Bergstrom DA, Kaneoke Y, Patel BN, Twery MJ, Walters JR (1999) Multisecond oscillations in firing rate in the basal ganglia: robust modulation by dopamine receptor activation and anesthesia. J Neurophysiol 81:2046-2055.

Sailer CA, Hu H, Kaufmann WA, Trieb M, Schwarzer C, Storm JF, Knaus HG (2002) Regional differences in distribution and functional expression of small-conductance $\mathrm{Ca}^{2+}$-activated $\mathrm{K}+$ channels in rat brain. J Neurosci 22:9698-9707.

Shen GY, Chen WR, Midtgaard J, Shepherd GM, Hines ML (1999) Computational analysis of action potential initiation in mitral cell soma and dendrites based on dual patch recordings. J Neurophysiol 82:3006-3020.

Shink E, Smith Y (1995) Differential synaptic innervation of neurons in the internal and external segments of the globus pallidus by the GABA- and glutamate-containing terminals in the squirrel monkey. J Comp Neurol 358:119-141.

Stiefel KM, Sejnowski TJ (2007) Mapping function onto neuronal morphology. J Neurophysiol 98:513-526.

Stiefel KM, Gutkin BS, Sejnowski TJ (2008) Cholinergic neuromodulation changes phase response curve shape and type in cortical pyramidal neurons. PLoS ONE 3:e3947.

Stiefel K, Gutkin BS, Sejnowski TJ (2009) The effects of cholinergic neuromodulation on neuronal phase-response curves of modeled cortical neurons. J Comput Neurosci 26:289-301.

Tateno T, Robinson HP (2007) Phase resetting curves and oscillatory stability in interneurons of rat somatosensory cortex. Biophys J 92:683-695.

Taylor AL, Goaillard JM, Marder E (2009) How multiple conductances de- termine electrophysiological properties in a multicompartment model. J Neurosci 29:5573-5586.

Terman D, Rubin JE, Yew AC, Wilson CJ (2002) Activity patterns in a model for the subthalamopallidal network of the basal ganglia. J Neurosci 22:2963-2976.

Tkatch T, Baranauskas G, Surmeier DJ (2000) Kv4.2 mRNA abundance and A-type $\mathrm{K}+$ current amplitude are linearly related in basal ganglia and basal forebrain neurons. J Neurosci 20:579-588.

Tsubo Y, Takada M, Reyes AD, Fukai T (2007) Layer and frequency dependencies of phase response properties of pyramidal neurons in rat motor cortex. Eur J Neurosci 25:3429-3441.

Urbain N, Gervasoni D, Soulière F, Lobo L, Rentéro N, Windels F, Astier B, Savasta M, Fort P, Renaud B, Luppi PH, Chouvet G (2000) Unrelated course of subthalamic nucleus and globus pallidus neuronal activities across vigilance states in the rat. Eur J Neurosci 12:3361-3374.

Vetter P, Roth A, Häusser M (2001) Propagation of action potentials in dendrites depends on dendritic morphology. J Neurophysiol 85:926937.

Wang J, Chen S, Nolan MF, Siegelbaum SA (2002) Activity-dependent regulation of HCN pacemaker channels by cyclic AMP: signaling through dynamic allosteric coupling. Neuron 36:451-461.

White JA, Netoff TI (2008) Synchronization in hybrid neuronal networks. In: Computational neuroscience in epilepsy (Soltesz I, Staley K, eds), pp 281-287. New York: Elsevier.

Xia XM, Fakler B, Rivard A, Wayman G, Johnson-Pais T, Keen JE, Ishii T, Hirschberg B, Bond CT, Lutsenko S, Maylie J, Adelman JP (1998) Mechanism of calcium gating in small-conductance calcium-activated potassium channels. Nature 395:503-507.

Yelnik J, Percheron G, François C (1984) A Golgi analysis of the primate globus pallidus. II. Quantitative morphology and spatial orientation of dendritic arborizations. J Comp Neurol 227:200-213. 